ARTICLE

\title{
Dominant role of CDKN2B/p15INK4B of 9p21.3 tumor suppressor hub in inhibition of cell-cycle and glycolysis
}

\author{
Yong Xia ${ }^{1,9}$, Yan Liu',9, Chao Yang ${ }^{2,9}$, Diane M. Simeone ${ }^{3,4}$, Tung-Tien Sun ${ }^{5}$, David J. DeGraff6,

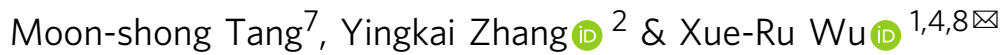

Human chromosome 9p21.3 is susceptible to inactivation in cell immortalization and diseases, such as cancer, coronary artery disease and type-2 diabetes. Although this locus encodes three cyclin-dependent kinase (CDK) inhibitors ( $15^{\mathrm{INK} 4 \mathrm{~B}}, \mathrm{p} 14^{\mathrm{ARF}}$ and $\mathrm{p} 16^{\mathrm{INK} 4 \mathrm{~A}}$ ), our understanding of their functions and modes of action is limited to the latter two. Here, we show that in vitro p15 ${ }^{\mathrm{INK} 4 \mathrm{~B}}$ is markedly stronger than p16 ${ }^{\mathrm{INK} 4 \mathrm{~A}}$ in inhibiting pRb1 phosphorylation, E2F activity and cell-cycle progression. In mice, urothelial cells expressing oncogenic HRas and lacking p15 INK4B, but not those expressing HRas and lacking p16 INK4A, develop early-onset bladder tumors. The potency of CDKN2B/p15 INK4B in tumor suppression relies on its strong binding via key $\mathrm{N}$-terminal residues to and inhibition of CDK4/CDK6. p15 INK4B also binds and inhibits enolase-1, a glycolytic enzyme upregulated in most cancer types. Our results highlight the dual inhibition of $\mathrm{p} 15^{\mathrm{INK} 4 \mathrm{~B}}$ on cell proliferation, and unveil mechanisms whereby $\mathrm{p} 15^{\mathrm{INK} 4 \mathrm{~B}}$ aberrations may underpin cancer and non-cancer conditions.

\footnotetext{
${ }^{1}$ Department of Urology, New York University School of Medicine, New York, NY, USA. ${ }^{2}$ Department of Chemistry, New York University, New York, NY, USA. ${ }^{3}$ Department of Surgery, New York University School of Medicine, New York, NY, USA. ${ }^{4}$ Department of Pathology, New York University School of Medicine, New York, NY, USA. ${ }^{5}$ Department of Cell Biology, New York University School of Medicine, New York, NY, USA. ${ }^{6}$ Department of Pathology and Laboratory Medicine and Department of Surgery, Division of Urology, The Pennsylvania State University College of Medicine, Hershey, PA, USA.

${ }^{7}$ Department of Environmental Medicine, New York University School of Medicine, New York, NY, USA. ${ }^{8}$ Veterans Affairs New York Harbor Healthcare System, New York, NY, USA. ${ }^{9}$ These authors contributed equally: Yong Xia, Yan Liu, Chao Yang. ${ }^{凶}$ email: xue-ru.wu@med.nyu.edu
} 
C

DKN2B gene resides on human chromosome 9p21.3 and encodes $\mathrm{p} 15^{\mathrm{INK} 4 \mathrm{~B}}$, a cell-cycle regulator that exhibits inhibitory activities toward cyclin-dependent kinases CDK4 and CDK6 (refs. ${ }^{1,2}$ ). Along with CDKN2A gene, which encodes $\mathrm{p} 14^{\mathrm{ARF}}$ and $\mathrm{p} 16^{\mathrm{INK} 4 \mathrm{~A}}$ via usage of separate first exons, and signals through p53 and pRb pathway, respectively ${ }^{3}$, these three CDK inhibitors arranged in tandem on $9 \mathrm{p} 21.3$ constitute a prominent tumor suppressor hub that is frequently deleted or epigenetically silenced prior to immortalization of primary cultured cells ${ }^{4-6}$ and in pre-tumor lesions ${ }^{7,8}$ and a multitude of tumor types ${ }^{3,4,9-11}$. Unlike p14 ${ }^{\mathrm{ARF}}$ and $\mathrm{p} 16^{\mathrm{INK} 4 \mathrm{~A}}$ whose tumorsuppressive activities have been well documented, $\mathrm{p} 15^{\mathrm{INK} 4 \mathrm{~B}}$ is often regarded as functionally equivalent to $\mathrm{p} 16^{\text {INK4A }}$ and a bystander during 9p21.3 deletion. These notions are, however, in stark contrast to the phenomena that (i) $\mathrm{p} 15^{\mathrm{INK} 4 \mathrm{~B}}$ can be targeted for deletion independently of $\mathrm{p} 14^{\mathrm{ARF}}$ and $\mathrm{p} 16^{\mathrm{INK} 4 \mathrm{~A} 2,12}$; (ii) the three CDK inhibitors can be down-regulated separately by promoter-specific methylation, cis-acting long non-coding antisense RNA (i.e., ANRIL and c-ANRIL) ${ }^{13-15}$ or divergent upstream signals (i.e., $\mathrm{p} 15^{\mathrm{INK} 4 \mathrm{~B}}$ by transforming growth factor beta (TGF-beta) ${ }^{16,17}$ ); (iii) p15 $5^{\text {INK4B }}$ becomes critically important in the absence of p16 ${ }^{\text {INK4A } 18}$; and (iv) in genetically engineered mice the loss of both p14 $4^{\mathrm{ARF}}$ and $\mathrm{p} 16^{\mathrm{INK} 4 \mathrm{~A}}$ fails to cooperate with oncogenes to elicit tumors in certain cell types, such as urothelium $^{19}$. By analyzing $\mathrm{p} 15^{\mathrm{INK} 4 \mathrm{~B}}$ in vitro, in vivo, and in human tumor cohorts, we present multiple lines of evidence here demonstrating $\mathrm{p} 15^{\mathrm{INK}} 4 \mathrm{~B}$ as a markedly stronger tumor suppressor than p16 $16^{\mathrm{INK} 4 \mathrm{~A}}$ via dual inhibition of cell cycle and aerobic glycolysis (Warburg effect) ${ }^{20}$. We provide detailed structural basis that sets $\mathrm{p} 15^{\mathrm{INK} 4 \mathrm{~B}}$ apart from $\mathrm{p} 16^{\mathrm{INK} 4 \mathrm{~A}}$ functionally and mechanisms whereby $\mathrm{p} 15^{\mathrm{INK} 4 \mathrm{~B}}$ exerts its potent activities. Our findings highlight a dominant role of $\mathrm{p} 15^{\mathrm{INK} 4 \mathrm{~B}}$ as a principal component of $9 \mathrm{p} 21.3$ locus in tumor suppression and the essential contributions of $\mathrm{p} 15^{\mathrm{INK} 4 \mathrm{~B}}$ deficiency to tumor initiation. Additionally, our study reveals an entry point through which to study the elusive molecular bases of non-cancer diseases, such as coronary artery disease, type 2 diabetes, and glaucoma, all of which are prominently associated with 9p21.3 aberrations ${ }^{9,21-23}$.

\section{Results and discussion}

Loss of $\mathrm{p} 15^{\mathrm{INK} 4 \mathrm{~B}}$ triggers early-onset and highly penetrant tumors from mouse urothelial cells expressing oncogenic HRas. Our initial motivation to investigate CDKN2B that encodes p15 INK4B (p15 hereon for brevity) was because of the high frequency (up to 70\%) of $9 \mathrm{p} 21.3$ loss in human urothelial tumors ${ }^{11,24-26}$, but the complete failure of loss of CDKN2A (encoding $\mathrm{p} 14^{\mathrm{ARF}}$ and $\mathrm{p} 16^{\mathrm{INK} 4 \mathrm{~A}}$ or $\mathrm{p} 14$ and p16, respectively, hereon) to cooperate with oncogenic HRas (Q61L) to induce any urothelial tumor in mice ${ }^{19}$. We hypothesized that, instead of p14 and p16, p15 might play a more important role in urothelial tumor suppression in the context of oncogenic HRas, and that p15 loss might be more functionally reflective of 9p21.3 loss. To test this, we crossed our Upk2-HRas transgenic mice, in which the expression of oncogenic HRas was driven by urothelium-restricted murine Upk2 promoter (Fig. 1a) ${ }^{19,27}$, with ubiquitous $C D K N 2 B$ knockout mice, in which exon 2 of $C D K N 2 B$ was replaced with a neo gene (Fig. 1a), which ablated p15 expression ${ }^{28}$. Intercrosses of the offspring yielded several hypothesis-testable genotypes (Fig. 1b), which we followed for up to 10 months for urothelial tumorigenesis (Fig. 1c-f). With dipstick test of spot urine from live mice as a preliminary screening for hematuria (Fig. 1c, arrow), a salient clinical manifestation in human bladder cancer patients ${ }^{29}$, we were able to identify potential bladder tumors that were primarily associated with $H R a s^{\mathrm{WT} / *} / \mathrm{p} 15^{-1-}$ genotype (Fig. 1c, upper panel, arrow). Gross anatomy of the urinary bladders from a cohort of mice sacrificed every 2 months beginning at 2 months of age $(n=$ 10/time point/genotype) revealed markedly enlarged bladders (Fig. 1c, lower panel (B as in bladder)) with significantly increased bladder weights (Fig. 1d, upper panel) in the $H R a s^{W T /^{*}} / p 15^{-/-}$ group, but not in any other groups)), even though the body weights did not differ significantly (Fig. 1d, lower panel). Hydronephrosis was noted only in the HRas ${ }^{\mathrm{WT} / *} / \mathrm{p} 15^{-/-}$group, particularly in the 4-month age group (Fig. 1c, lower panel ( $\mathrm{K}$ as in kidney)), due likely to bladder tumor overgrowth and blockage of the urinary tract outlet. Overall, in $H R a s^{\mathrm{WT}} / * / \mathrm{p}^{*} 5^{-1-}$ mice, bladder tumors were confirmed microscopically in $20 \%$ of the mice by 2 months, $80 \%$ of the mice by 4 months, and $100 \%$ of the mice by 6 months (Fig. 1e, f). Bladder tumors were also detected in HRas $^{\mathrm{WT} / *} / \mathrm{p} 15^{\mathrm{WT} /-}$ mice (e.g., heterozygous p15 deletion), albeit at a much lower frequency ( $30 \%$ by 10 months), later onset (beginning at 4 months) and smaller sizes (visible only microscopically). While a relatively small number of $H R a s^{\mathrm{WT}} / *$ / $p 15^{+/-}$(in which p15 was heterozygous) also developed bladder tumors, the tumor cells retained one p15 allele. We presently cannot rule out whether the remaining allele is inactivated by hypermethylation, ANRIL, or inactivation of the upstream regulators, such as TGF- $\beta$ (see later). This is an important question that warrants further investigation. By histology, the $\mathrm{p} 15^{-1-}$ mice exhibited normal-appearing urothelium and the $H R a s^{\mathrm{WT}} /^{*}$ mice urothelial hyperplasia (Fig. 1f). In contrast, the $H R a s^{\mathrm{WT} / *} / \mathrm{p} 15^{-I-}$ mice harbored bladder tumors that were consistently papillarylooking, low pathological grade, and confined to the urothelial layer without breaching the basement membrane, thus being lowgrade, non-invasive tumors (Fig. 1f). These data provide the first in vivo experimental evidence indicating that p15 loss and HRas activation are two highly cooperative events that are necessary and sufficient to initiate low-grade, non-invasive bladder tumor, the most prevalent, recurrent and expensive type of bladder tumor in human patients ${ }^{30-32}$.

p15 is a markedly more potent urothelial tumor suppressor than p16 in vivo. Although we previously showed that the loss of CDKN2A failed to cooperate with oncogenic HRas to induce urothelial tumors ${ }^{19}$, and we showed here that the loss of p15 and oncogenic HRas together are highly tumorigenic in urothelial cells (Fig. 1), these two studies were done at different time periods and settings. Additionally, since CDKN2A encodes both p16 and p14 (p19 in mice), it was necessary that we ascertain under the same experimental condition the relative potency of p15 versus p16 (instead of p16 and p19 together) in urothelial tumor suppression as well as the in vivo effects of their loss in urothelial tumor initiation. With this in mind, we carried out crosses between Upk2-HRas* transgenic mice and p16 knockout mice in which exon $1 a$ of $C D K N 2 A$ gene was replaced with a neo gene, which inactivates p16 but not alternatively transcribed p19 (Fig. 2a and ref. ${ }^{33}$ ). In parallel, we carried out de novo crosses between Upk2-HRas* transgenic mice and the aforementioned p15 knockout mice (Fig. 2a). From these crosses we obtained a number of different genotypes, but chose to focus on several genotypes that were most germane to define the in vivo differences between p16 deficiency and p15 deficiency in urothelial tumorigenesis, i.e., (i) wild-type (WT) mice that exhibit normal urothelium; (ii) single-allele HRas ${ }^{W T / *}$ mice which we previously showed to consistently develop simple urothelial hyperplasia before 10 months of age ${ }^{19}$; (iii) $H R a s^{\mathrm{WT} / *} / p 15^{-/-}$mice; and (iv) HRas ${ }^{\mathrm{WT} / *} / p_{16^{-l-}}$ mice (Fig. 2b). Western blotting verified the absent expression of p15 and p16 in the urothelial cells of the aforementioned group (iii) mice and group (iv) mice, respectively (Fig. 2c). Interestingly, there was an induction of p15 in HRas*expressing urothelial hyperplasia, and more so in HRas*. 
a
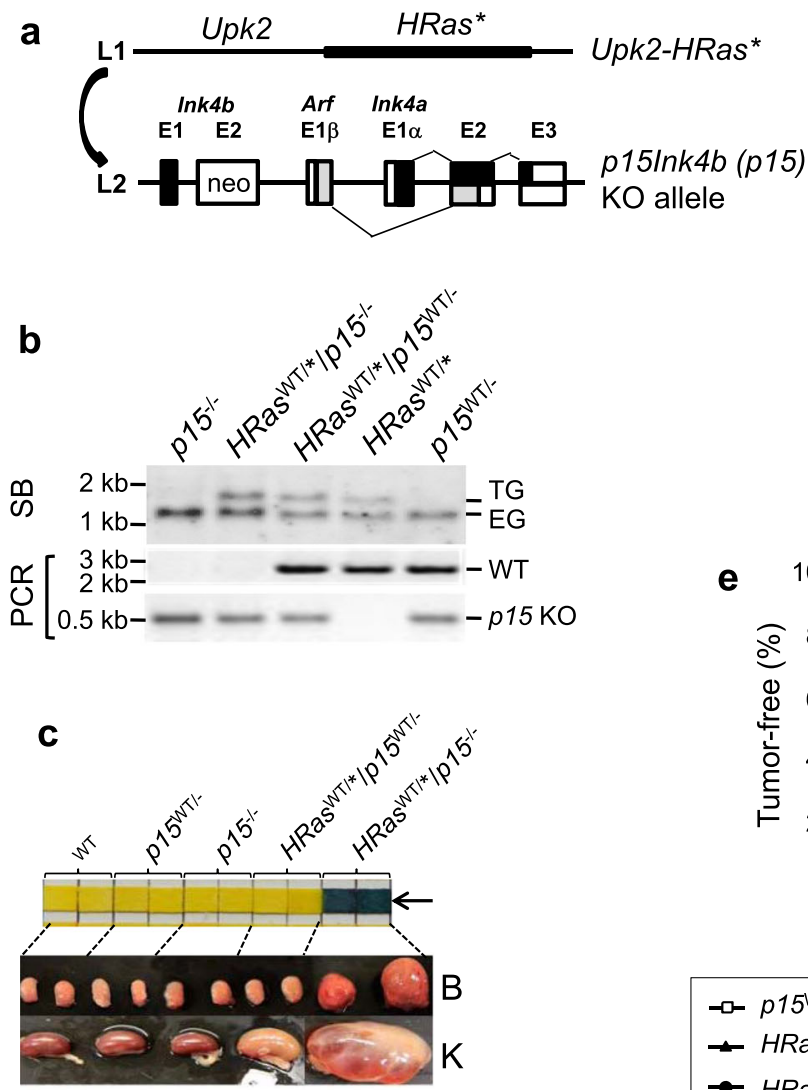

d
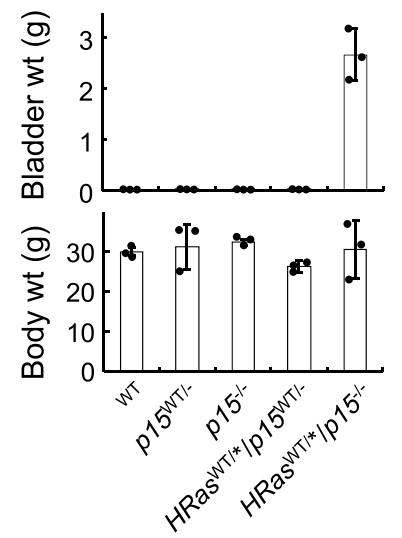

e

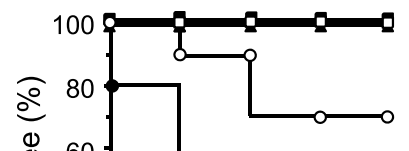

$P=1.5 E-4$

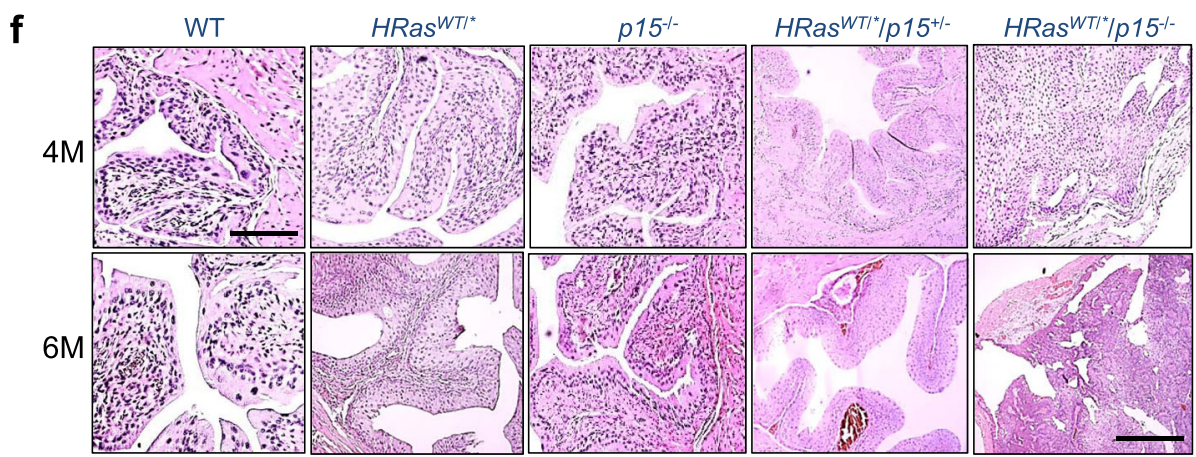

Fig. 1 Loss of p15 in mouse urothelial cells expressing oncogenic HRas triggers early-onset and highly penetrant urothelial carcinomas that strongly resemble low-grade, papillary, non-muscle-invasive bladder cancer in humans. a Mouse breeding scheme. Transgenic mice in which oncogenic $H R a s$ expression was under the control of murine Upk2 promoter (abbreviated as HRas WT/ ${ }^{*}$ mice) was crossed and backcrossed to $p 15 / n k 4 b$ knockout (p15 ${ }^{-}$) mice in which exon 2 of the gene was replaced by a neo gene, which ablates p15 expression ${ }^{53}$. b Representative genotypes from one litter of the aforementioned crosses. SB Southern blotting detecting the restriction enzyme-digested transgene fragment (TG) of Upk2-HRas, and endogenous Upk2 fragment (EG); and PCR detecting the wild type (WT) and the p15 knockout (KO) allele. Genotyping was performed on every mouse with highly reproducible results, and cumulatively $n>50 /$ genotype. Source data are provided as a Source Data file. c Urine dipstick test of hematuria (upper panel; only the bloodtesting row is shown) and gross anatomy of bladder (B) and kidney (K) (lower panel) of 4-month-old mice (2 mice/genotype shown). Color change to dark blue (arrow) indicates hematuria. Note the highly enlarged renal pelvis in the HRas WT/ $/ \mathrm{p} 15^{-/-}$mice. The kidney of the $H R a s^{W T /{ }^{*} / p 15^{W T} /-}$ mouse was slightly bigger but lacked hydronephrosis upon sectioning. $\mathbf{d}$ Bladder and body weights (wt) of mice sacrificed at 6 months of age ( 3 mice/genotype). Data are presented as mean \pm SD. e Tumor-free rate for genotypes indicated in the box (10 mice/time point/genotype). $P$ value indicated $(P=1.5 \mathrm{E}-4)$ was for comparison between $H \operatorname{Ras}^{W T /{ }^{*}} / \mathrm{p} 15^{\mathrm{WT} /-}$ mice and $\mathrm{HRas} \mathrm{WT}^{*} / \mathrm{p} 15^{-/-}$mice, using the Kaplan-Meier method followed by log-rank statistical test using software SPSS v17.0. $\mathbf{f}$ Representative H\&E images of mouse bladders from the key genotypes at 4 and 6 months of age. $n=10 /$ genotype/time point. All the panels are of the same magnification with the bar in the upper-left panel equaling to $100 \mu \mathrm{m}$, except the lower right panel (scale bar $=500 \mu \mathrm{m}$ ).

expressing and p16-lacking urothelial cells. While p16 was not significantly induced in HRas*-expressing urothelial cells, deletion of p15 from these cells coincided with a strong induction of p16 (Fig. 2c). It appeared therefore that oncogenic HRas triggered a reciprocal compensatory induction of p15 and p16 in urothelial cells when the other protein is absent, albeit with divergent potencies in tumor suppression (see later). Finally, there was a strong increase of p19 expression that was confined to HRas*expressing and p15-lacking urothelial cells (Fig. 2c). However, such an increase clearly did not prevent urothelial tumor 

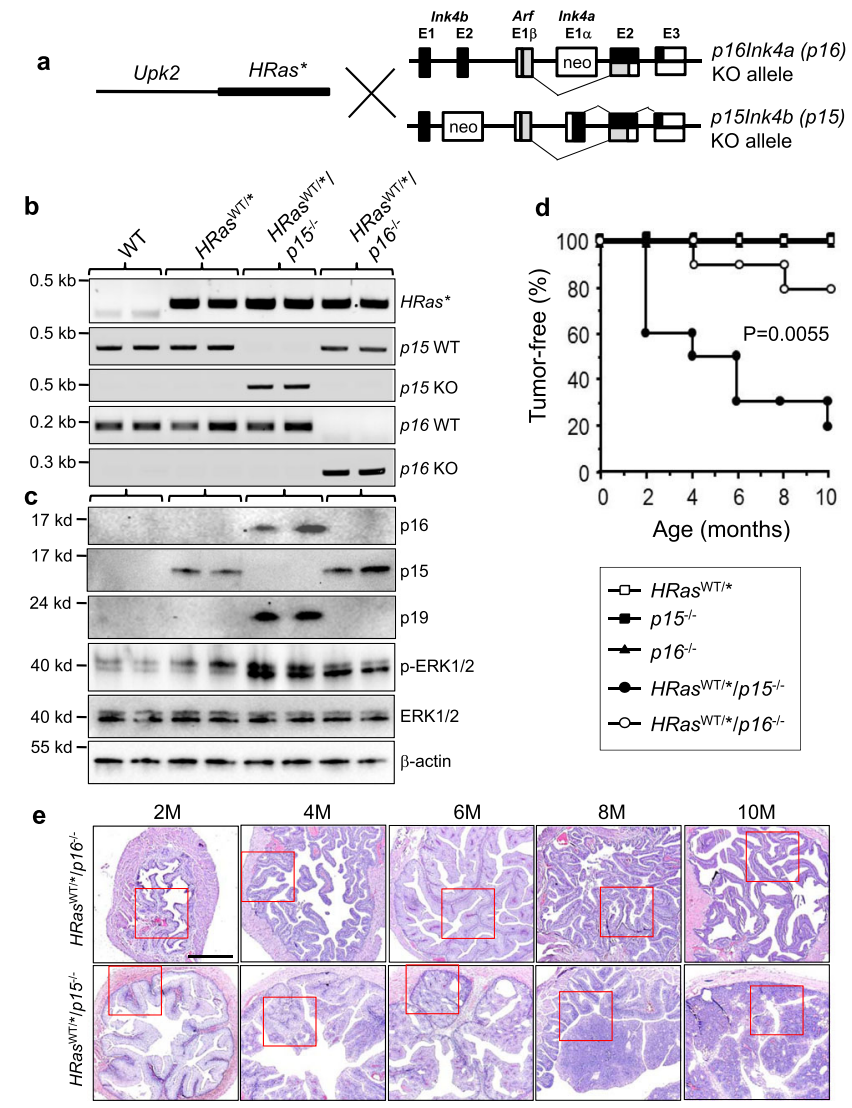

Fig. 2 Divergent effects of p15 loss or p16 loss in HRas-mediated urothelial tumorigenesis. a Mouse breeding scheme. Two sets of intercrosses were carried out (i) between Upk2-HRas transgenic mice (HRas $\left.{ }^{\star}\right)$ and p16lnk4a (p16) knockout ( $\left(16^{-/-}\right)$in which exon $1 \alpha$ of the gene was replaced by the neo gene, thus leaving an independent transcript of the gene, p19Arf, intact; and (ii) between HRas ${ }^{\star}$ transgenic mice and p15/nk4b (p15) knockout ( $p 15^{-/-}$) mice. b Representative genotyping results by genomic PCR of major experimental groups and controls (1 mouse/lane; 2 mice/genotype). The genotyping was performed on every mouse with highly reproducible results. c Western blotting of p16, p15, p19, ERK1/2 and phosphorylated ERK1/2, and $\beta$-actin (loading control) in urothelial cells of specific genotypes. Each lane represents one mouse and two independent mice were used per genotype. The experiment was repeated twice with similar results. Source data are provided as a Source Data file. d Divergent tumorigenesis in urothelial cells expressing HRas ${ }^{\star}$ and lacking p16 versus those expressing HRas* and lacking p15 ( $n=10 /$ time point/genotype). $P$ value shown resulted from the comparison between $H$ Ras $\mathrm{WT} / /^{*} / \mathrm{p} 16^{-/-}$and HRas $\mathrm{WT}^{*} / \mathrm{p} 15^{-/-}$mice, using the Kaplan-Meier method followed by logrank statistical test by software SPSS v17.0. Note the markedly earlier onset and higher frequency of tumorigenesis in $\mathrm{HRas} W T /{ }^{*} / \mathrm{p} 15^{-/-}$mice than in $H$ Ras $\mathrm{WT} /{ }^{*} / \mathrm{p} 6^{-/-}$mice. Also note that, while the significant induction of p16 in p15-lacking mice did not prevent bladder tumor formation, the significant induction of p15 in p16-lacking mice did exert a strong tumor-suppressive effect (Fig. 1d). e Bladder tumorigenic status in HRas WT/ $/ \mathrm{p}^{*} 6^{-/-}$versus HRas WT/*/p15-/- mice. Representative images of H\&E-stained crosssections of bladders from mice (age 2-10 months) expressing oncogenic HRas and lacking p16 or p15 as shown in the cohort in Fig. 1d. Note that the HRas $\mathrm{WT}^{*} / \mathrm{p} 15^{-/-}$mice developed nodular urothelial hyperplasia at 2 months of age and superficial papillary bladder tumors from 4 months onward, and that in contrast, the bladders of the HRas $\mathrm{WT} /{ }^{*} / \mathrm{p} 16^{-/-}$mice only contained mucosa folds from simple urothelial hyperplasia, and were largely devoid of tumors (see corresponding high-powered images for redboxed areas in Supplementary Fig. 1). All panels are of the same magnification and the scale bar in the upper-left panel equals $500 \mu \mathrm{m}$. $n=10$ mice per genotype per time point. formation. Whether this prevented urothelial tumor progression, i.e., from the non-invasive to the invasive stage, requires further investigation.

Tumorigenesis analysis showed that $\mathrm{HRas}^{W T / *}$ mice were devoid of any urothelial tumors, and that only 10 and $20 \%$ of HRas $^{\mathrm{WT} / *} / p 16^{-1-}$ mice developed bladder tumors by 6 and 8 months of age, respectively (Fig. 2d), that could only be visualized microscopically. In contrast and general support of our initial cohort shown in Fig. 1, bladder tumors arose in $40 \%$ of HRas $^{\mathrm{WTT} / *} / p 15^{-1-}$ mice as early as 2 months, in $70 \%$ of the mice by 6 months and in $80 \%$ of the mice by 10 months (Fig. $2 d$, e and Supplementary Fig. 1). Without exception, these tumors were of low pathological grade, superficially located and papillary-looking (Fig. 2e; for higher magnification, see Supplementary Fig. 1). To further establish the different potencies of p15 versus p16 in tumor suppression in the context of HRas* mutation, we analyzed yet another cohort of mice (Supplementary Fig. 2). The data were highly reproducible in that, by 3 months of age, $40 \%$ of the HRas $\mathrm{WT}^{*} / p 15^{-1-}$ mice developed urothelial tumors, whereas none of $H R a s^{\mathrm{WT} / *} / p 16^{-/-}$did the same. We do recognize the slight differences in tumor onset and frequencies in our HRas ${ }^{\mathrm{WT}} /^{*} / \mathrm{p} 15^{-1-}$ mice between Cohort 1 (Fig. 1e) and Cohorts 2 (Fig. 2d) and 3 (Supplementary Fig. 2). We believe that small differences in genetic background, i.e., Cohort 1 mice were closer to C57BL/6 and Cohort 2 and 3 mice were closer to into $\mathrm{FVB} / \mathrm{N}$, might contribute to those differences.

While the tumor cells in the HRas $\mathrm{WT} / * / p 15^{-/-}$mice continued to express urothelial markers, such as Upk $3 \mathrm{a}^{34}$, they diminished in intensity and were accompanied by a significant increase of markers indicative of cell proliferation, such as Ki67, or urothelial tumor progenitor cells, such as Krt5 and Krt14 (Fig. 3a) 35,36 . The opposite was true in $H$ Ras ${ }^{\mathrm{WT} / *} / p 16^{-I-}$ mice (Fig. 3a). These data strongly suggest that, during HRas*mediated urothelial tumorigenesis, p15 loss plays a much more critical role in bladder tumor initiation than p16 loss. Furthermore, while we observed a reciprocal induction of p15 and p16 in the absence of the other protein (Fig. 2c), deletion of both p15 and p16 in $H R a s^{\mathrm{WT} /{ }^{*}} / \operatorname{Ink} 4 a b^{-/-}$mice did not significantly increase the tumor frequency or shorten the tumor latency in two independent cohorts (Supplementary Fig. 3) beyond what was seen in $\operatorname{HRas}^{\mathrm{WT} / *} / p 15^{-/-}$mice (Figs. 1e, $\mathrm{f}$ and $2 \mathrm{~d}$, e and Supplementary Figs. 1 and 3 ). This further supports the idea that p15 loss is functionally more important than p16 loss in HRas*-mediated urothelial tumorigenesis, and that loss of both p16 and p15 does not appear synergistic or significantly additive in urothelial tumor initiation. Nevertheless, further investigation with large cohorts and more time points is necessary to firmly establish this conclusion.

p15 is significantly more inhibitory of cell-cycle progression and proliferation than p16. To understand the mechanisms underlying the different potencies of p15 and p16 in urothelial tumor suppression, we examined cell-cycle distribution by fluorescence-activated cell sorting (Fig. 3b) and pRB1 phosphorylation by western blotting (Fig. 3c) in the in vivo mouse cohort as shown in Fig. 2d, e and Supplementary Fig. 1. The fraction of urothelial cells in $\mathrm{G} 0+\mathrm{G} 1$ phases was much lower and the fraction in $\mathrm{S}$ and $\mathrm{G} 2 / \mathrm{M}$ phases much higher in $\mathrm{HRas} \mathrm{WT}^{\mathrm{W}} / \mathrm{p} 15^{-/-}$mice than in $\mathrm{HRas}^{\mathrm{WT} / *} / \mathrm{p}^{*} 6^{-1-}$ mice (Fig. 3b). This corresponded well with the elevated phosphorylation of pRB1 at both S780 and S807/ 811 sites in $\mathrm{HRas}^{\mathrm{WT} / *} / \mathrm{p} 15^{-1-}$ mice (Fig. 3c), suggesting that the lack of p15 reduced the inhibition on CDK4 and/or CDK6 significantly more so than the lack of p16.

To independently verify the in vivo findings, we examined the potency of $\mathrm{p} 15$ and p16 on growth inhibition by restoring their 


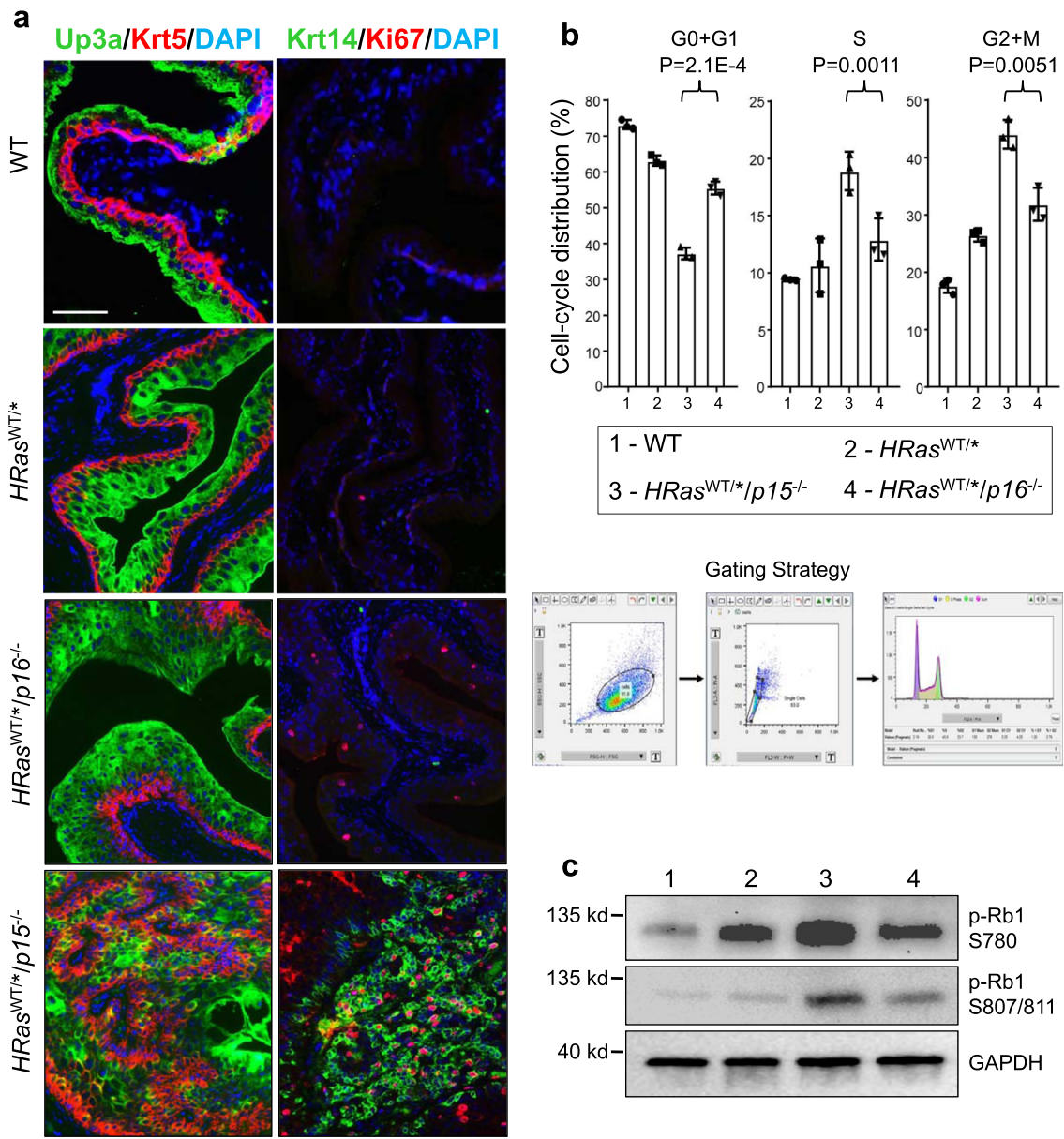

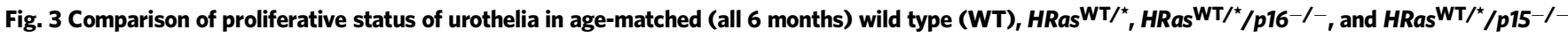
mice. a Immunofluorescent staining of urothelial differentiation (Upk3a), proliferation (Ki67), and bladder cancer progenitor cell (Krt5 and Krt14) markers. Note the significantly greater $\mathrm{Krt5}, \mathrm{Krt} 14$, and $\mathrm{Ki} 67$ positive urothelial cells in $H$ Ras WT/ ${ }^{\star} / \mathrm{p} 15^{-/-}$mice than in $H$ Ras $\mathrm{WT}^{*} / \mathrm{p} 16^{-/-}$mice. $n=5 \mathrm{per}$ genotype indicated on the left. The panels are of the same magnification and the bar in the upper-left panel equals $50 \mu \mathrm{m}$. b Cell-cycle distribution ( 3 mice/ genotype). Freshly stained urothelial cells by propidium iodine underwent (see Gating Strategy) (i) forward versus side scatter to remove small debris and large aggregates, (ii) width versus area to remove doublets, and (iii) cell-cycle distribution based on DNA content using FlowJo-embedded cell-cycle analysis. Data are presented as mean value \pm SD. Two-sided $t$-tests were performed to compare the significance of the differences between the two groups (HRas $\mathrm{WT} /{ }^{*} / \mathrm{p} 15^{-/-}$versus HRas $\mathrm{WT} /{ }^{*} / \mathrm{p} 16^{-/-}$). The $P$ values are shown in the figure. Note that urothelial cells in $\mathrm{S}$ and $\mathrm{G} 2+\mathrm{M}$ phases were $2-3-$ fold higher

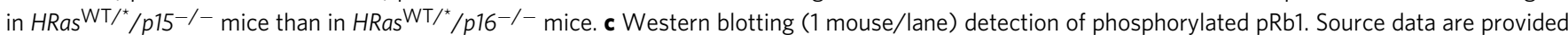
as a Source Data file. Note that the pRb1 phosphorylation (at both $\mathrm{S} 780$ and S807/811) was significantly higher in HRas WT/*/p15-/- mice than in

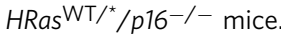

expression in two human bladder cancer cell lines (UMUC3 and RT112) that had homozygous deletion of 9 p21.3 (ref. ${ }^{37}$ ) and therefore lacked endogenous p15 and p16. By first stably transfecting the cell lines with CMV-driven reverse tetracycline transactivator (CMV-rtTA) and then stably transfecting with tetracycline response element (TRE)-driven, Myc-tagged p15 cDNA or TRE-driven, Myc-tagged p16 cDNA, we were able to use tetracycline to inducibly express, detect and semi-quantify, via the Myc-tagged, restored p15 or p16. We found p15 to be significantly more inhibitory of cell proliferation than p16 in both cell lines (Fig. 4a). Additionally, compared with p16, p15 more significantly increased the fractions of cells in $\mathrm{G} 0+\mathrm{G} 1$ phases and decreased the fractions in $\mathrm{S}$ and $\mathrm{G} 2+\mathrm{M}$ phases (Supplementary Fig. 4a). Furthermore, p15 was significantly more inhibitory of pRB1 phosphorylation at $S 780$ and $S 807 / 811$ than p16, whereas neither p15 nor p16 affected the phosphorylation of other $\mathrm{Rb}$ family members pRB2 and p107 (Fig. 4b). Consistent with our results on reduced pRB1 phosphorylation by p15 expression which should lead to increased pRB1/E2F binding and decreased
E2F release, the overall E2F activity was much lower in p15expressing cells than in p16-expressing cells (Supplementary Fig. $4 b)$.

p15 binds more efficiently to CDK4 and CDK6 than p16. Because CDK inhibitors inhibit CDKs via direct binding ${ }^{38,39}$, we tested whether p15 bound to CDK4 and CDK6 more efficiently than p16 using two independent approaches. In the first, we inducibly expressed Myc-tagged p15 or Myc-tagged p16 in UMUC3 cells, and carried out immunoprecipitation using an anti-Myc antibody, followed by western blotting detection and semi-quantification of CDK4 and CDK6 that naturally bound to p15 or p16. When normalized to immunoprecipitated Myctagged p15 and Myc-tagged p16 and then to input, p15 immunoprecipitated roughly sixfold and fivefold of CDK4 and CDK6, respectively, compared to p16 (Fig. 4c). In the second approach, we performed a pull-down experiment of freely available CDK4 and CDK6 in the total protein extracts by expressing 

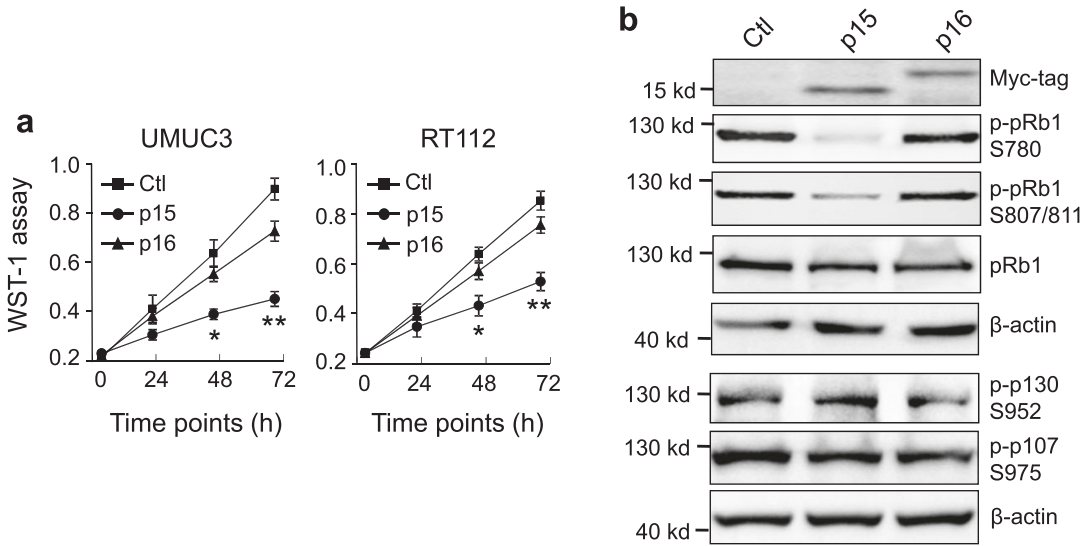

C

d
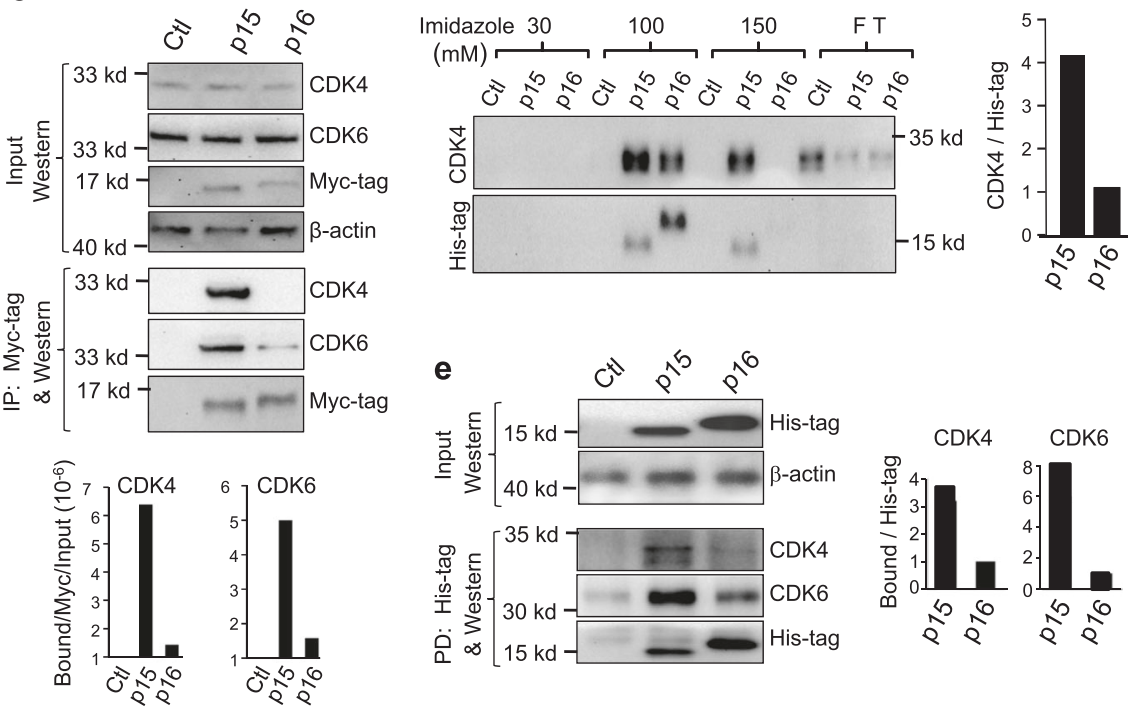

Fig. 4 Greater inhibition of proliferation by p15 than by p16 via their differential binding to CDK4 and CDK6. a Human bladder cancer cell lines, UMUC3 and RT112, both lacking endogenous p15 and p16, were stably transfected and induced to express either protein, using a two-vector, tetracycline-inducible system, followed by proliferation (WST-1 assay) at the indicated post-induction time points. Cell number was normalized by western blotting (as shown in $\mathbf{b}$ ). In $\mathbf{a} n=4$ of biologically independent samples. Data are presented as mean value \pm SD. Two-sided $t$-test was performed to compare the significance of the difference between the two groups (p15 versus p16). The $P$ values are: for UMUC3: $\left({ }^{\star}\right), 1.3 \mathrm{E}-4 ;\left({ }^{\star}\right)$, 3.5E-5; and for RT112: $\left(^{\star}\right)$ 0.0019 and $\left({ }^{\star \star}\right) 8.8 \mathrm{E}-5$. Note only a relatively small inhibition of proliferation and cell-cycle progression by p16 expression and a much greater inhibition by p15 expression. $\mathbf{b}$ Western blotting of key cell-cycle regulators of transfected UMUC3 cells analyzed $24 \mathrm{~h}$ after the induced expression of p15 or p16, showing the marked inhibition of pRb1 phosphorylation by $\mathrm{p} 15$ and much less so by p16, and that p15 did not affect the phosphorylation of other pRb family proteins p130 or p107. $n=3$ per genotype indicated at the top. c-e Differential binding of p15 and p16 to CDK4 and CDK6. c Immunoprecipitation. UMUC3 cells were stably transfected and induced by tetracycline to express Myc-tagged p15 or Myc-tagged p16, followed by immunoprecipitation using anti-Myc-tag antibody and then western blot detection of co-immunoprecipitated proteins. The lower panels were representative semi-quantitation by densitometry of immunoprecipitated CDK4 and CDK6 by p15 or p16 in reference to the Myc-tag (middle panels) and input (top panels). The experiments were repeated three times with similar results. Note that, with equivalent amounts, p15 immunoprecipitated significantly more CDK4 and CDK6 than p16. d Pull-down assay. Histidine-tagged p15 or p16 produced by recombinant $E$. coli were immobilized on nickel columns and incubated with total protein extracts from untransfected, parental UMUC3 cells. Bound proteins were eluted by increasing concentrations of imidazole and detected by western blotting. The right panel was a representative semiquantitation by densitometry of pulled-down CDK4 by p15 or p16 at $100 \mathrm{mM}$ imidazole in reference to the His-tag. The experiments were repeated twice with similar results. Note that, proportionally, with $100 \mathrm{mM}$ imidazole, p15 pulled down significantly greater amounts of CDK4 than p16. e An independent experiment similar to that described in $\mathbf{d}$, showing greater amounts of CDK4 and CDK6 were pulled down by p15 than by p16. The right panels were representative semi-quantitation by densitometry of pulled-down CDK4 and CDK6 by p15 or p16 in reference to the His-tag. The experiments were repeated twice with similar results. Source data are provided as a Source Data file.

recombinant, histidine-tagged p15 or histidine-tagged p16 in $E$. coli, conjugating either protein to nickel columns, and incubating them with total proteins extracted from untransfected, parental UMUC3 cells. When referenced to histidine-tagged p15 and histidine-p16 eluted by imidazole at $100 \mathrm{mM}$, over fourfold more CDK4 was eluted with p15 than with p16 (Fig. 4d). This result was confirmed by another independently performed experiment, showing about four- and eightfold enrichment of CDK4 and
CDK6, respectively, with p15 as a bait, compared to p16 as a bait (Fig. 4e).

The $\mathrm{N}$ terminus of p15 mediates the strong binding of p15 to CDK4 and CDK6. p15 and p16 are highly homologous (69\% identity in amino acid sequences) with the exception of their $\mathrm{N}$ termini $(\mathrm{N})$ and the longer $\mathrm{C}$ terminus $(\mathrm{C})$ unique to p16. To discern the region responsible for the strong interaction between 

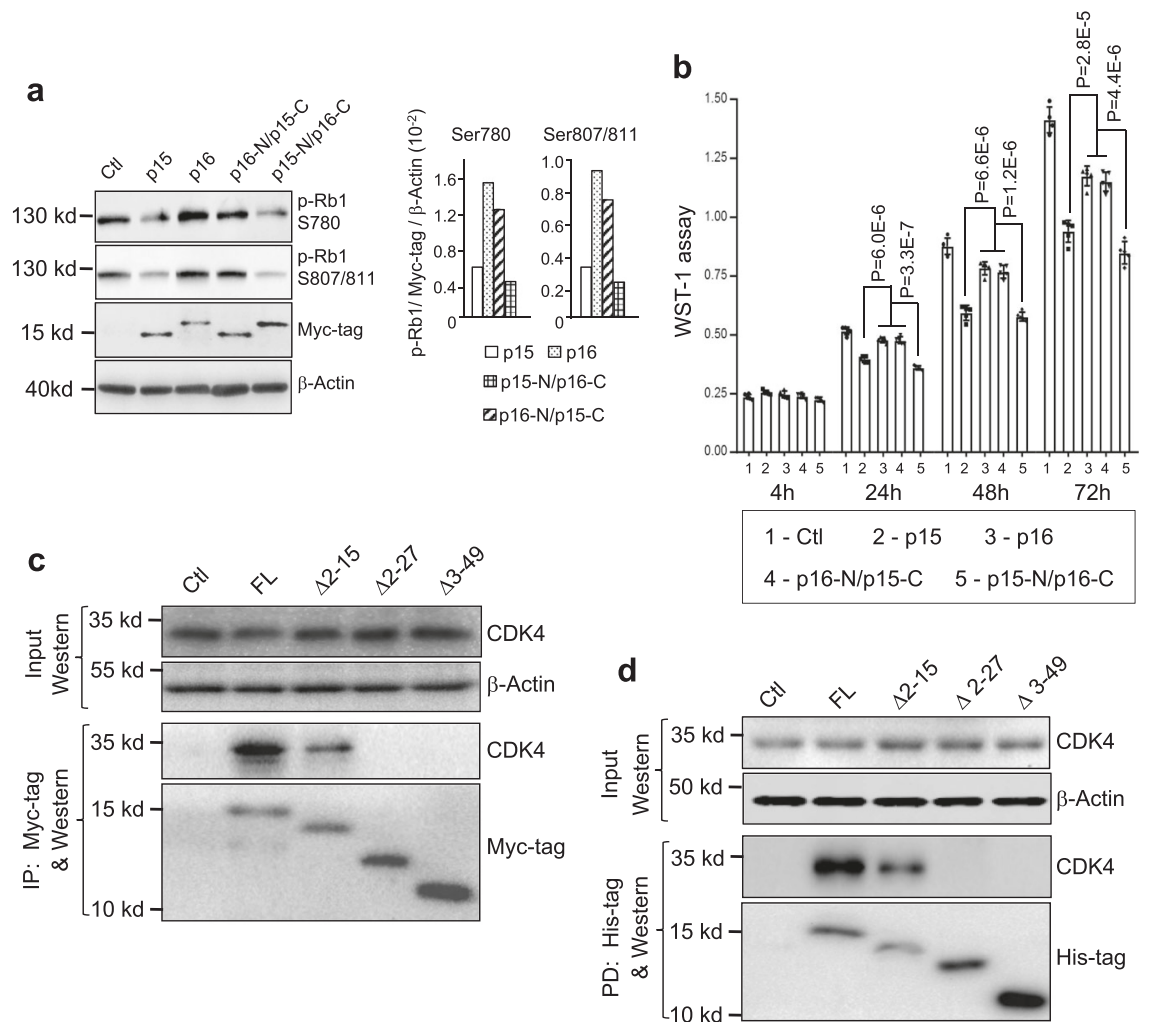

Fig. 5 Critical role of the $\mathbf{N}$ terminus of p15 in binding to and inhibiting CDK4 and CDK6. $\mathbf{a}$, $\mathbf{b}$ Domain switching between p15 and p16 narrows strong growth inhibitory activity of p15 to its $\mathrm{N}$ terminus. Mammalian expression vectors bearing Myc-tagged cDNA hybrids (i.e., p16-N terminus (p16-N) plus p15-C terminus ( $15-C)$ ) or p15-N terminus ( $15-N$ ) plus p16-C terminus ( $16-C)$ ), along with control vectors (CtI) bearing Myc-tagged wild-type p15 or wild-type p16 were stably transfected and inducibly expressed in UMUC3 cells. pRb1 phosphorylation status $24 \mathrm{~h}$ after induced expression of wild type and hybrid proteins (a) and cell proliferation with time course indicated (b) were assessed by western blotting with semi-quantitation and WST-1 assay, respectively (a, right panels), were representative semi-quantitation by densitometry of the phosphorylated pRb1 shown in the left panel in reference to Myc-tag and $\beta$-actin. The experiments were repeated twice with similar results. $\mathbf{b}$ p16 and p16-N/p15-C were statistically compared as one group because of their highly similar readings and compared with p15 and p15-N/p16-C as individual groups. Note that p15-N/p16-C was as effective as wild-type p15 in inhibiting pRb1 phosphorylation and cell proliferation and, in comparison, that p16-N/p15-C was as ineffective as wild-type p16 in inhibiting pRb1 phosphorylation and cell proliferation. $n=5$ of biologically independent samples. Data are presented as mean \pm SD. Two-sided $t$-test was performed to compare the significance of the difference between the two groups (p15 versus p16; and p16-N/p15-C versus p15-N/p16-C). The $P$ values are shown in the figure. c, d N-terminal deletion of p15 further narrows the critical region for CDK4 binding. Mammalian expression vectors bearing Myc-tagged deletion mutants of p15 cDNA as indicated above each panel, along with mock vector control (CtI) and full-length (FL) p15 cDNA were stably transfected and inducibly expressed in UMUC3 cells. Immunoprecipitation (IP) followed by western blotting was then carried out (c). Alternatively, the histidine-tagged mutants were expressed in E. coli, immobilized on nickel column and incubated with total protein extracts from parental UMUC3 cell line (pull-down experiment, PD), followed by western blotting (d). Note that in both experiments the deletion mutant $\Delta 2-15$ reduced binding to CDK4 and the deletion mutants $\Delta 2-27$ and $\Delta 3-49$ completely abolished its binding ability to CDK4. Experiments in $\mathbf{c}$ and $\mathbf{d}$ were repeated three times with similar results.

p15 and CDK4/CDK6, we first performed domain switch between p15 and p16 by engineering p15/p16 hybrids, i.e., p15-N/p16-C and p16-N/p15-C. Transfection and inducible expression of Myctagged hybrids and their wild-type controls in p15/p16-lacking, parental UMUC3 cells showed that p15-N/p16-C was as effective as wild-type p15 in inhibiting pRB1 phosphorylation (Fig. 5a) and cell proliferation (Fig. 5b). In contrast, p16-N/p15-C was as ineffective as wild-type p16 in inhibiting pRB1 phosphorylation (Fig. 5a) and cell proliferation (Fig. 5b). Step-wise N-terminal deletion followed by transfection and immunoprecipitation (Fig. 5c) or by production of deletion mutants in E. coli and pulldown experiments (Fig. 5d) further showed that the deletion of the first 15 amino acids significantly reduced the binding of p15 to CDK4, and that the deletion of the first 27 amino acids completely abolished the binding of p15 to CDK4. These results established the critical importance of the $\mathrm{N}$ terminus of p15 in conferring its strong ability to bind CDK4 and CDK6 and inhibit pRb1 phosphorylation, cell-cycle progression, and proliferation.
Key salt-bridges and hydrogen-bonds between p15 and CDK6 underlie strong binding. Because the crystal structure of p15 is unavailable, we made use of the available structure of cocrystallized p16-CDK6 $6^{40}$ for computational modeling and molecular dynamics (MD) simulations for p15-CDK6 interaction (Fig. 6a, b, Supplementary Fig. 5 and Supplementary Table 3). The simulation results suggested several key interactive points between the $\mathrm{N}$ terminus of p15 and CDK6, particularly in the salt-bridges and $\mathrm{H}$ bonds (Fig. 6a). Specifically, the $\mathrm{N}$-terminal residues Ser20 and Arg24 of p15 could form $\mathrm{H}$ bonds and saltbridges with Glu18 and Glu21 of CDK6 (Fig. 6a). Additionally, Glu16 of p15 could form salt bridge with Arg45 of p15, which could stabilize Arg45 in a position to form additional salt bridge with Glu18 of CDK6 (Fig. 6a). These interactions could cooperate with each other to form a relatively stable network as suggested by $\mathrm{MD}$ simulations (Fig. 6a). In contrast, from both the p16-CDK6 crystal structure and p16-CDK6 MD simulations, p16 lacked such N-terminal interactions with CDK6 (Fig. 6a). 
$\mathbf{a}$
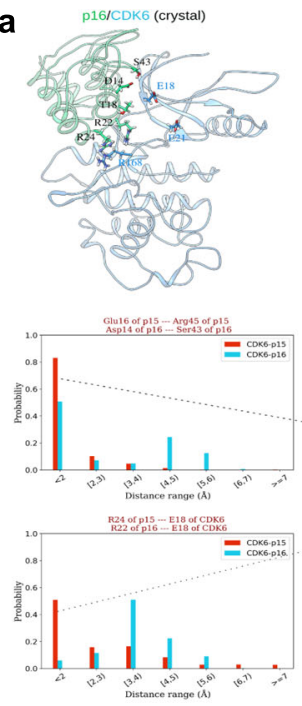
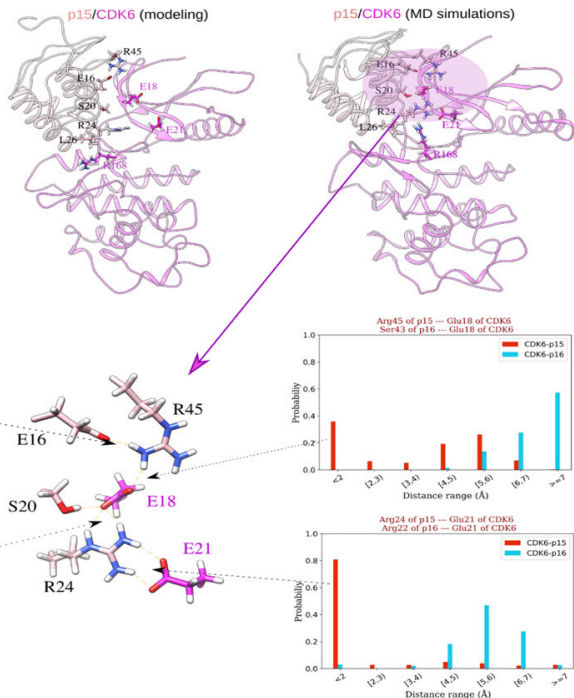

b
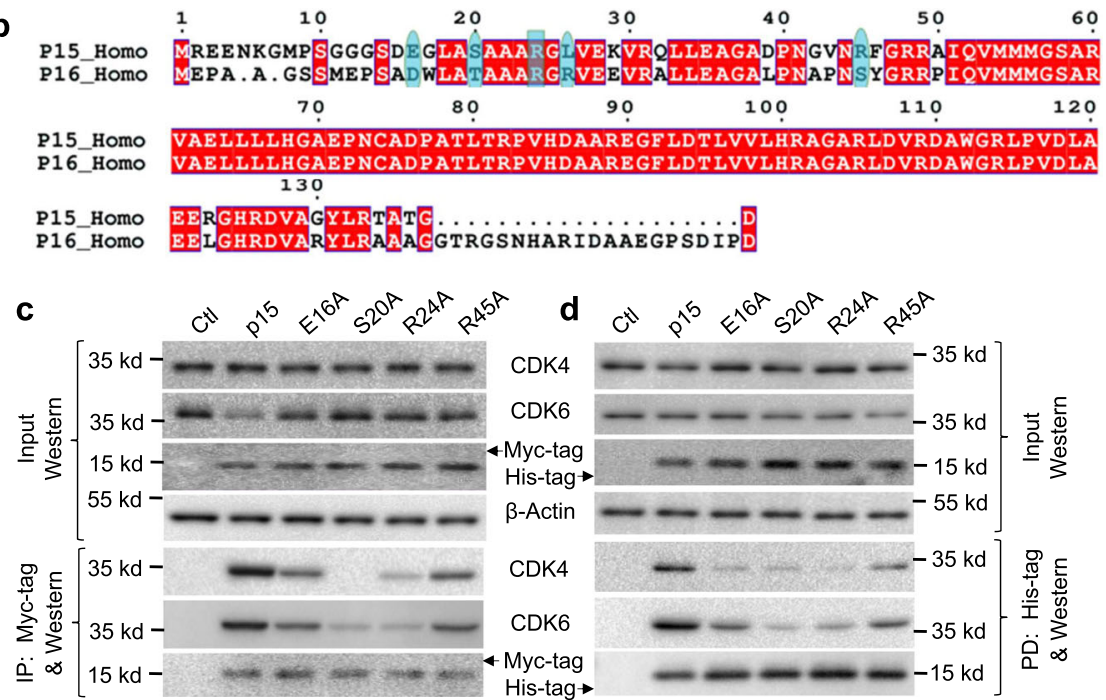

Fig. 6 Structural modeling and experimental verification of amino acids in p15 that are critical for binding to CDK6. a Homology modeling of p15 binding to CDK6 (top middle panel) based on the available crystal structure of p16/CDK6 complex (top left panel). Molecular dynamics simulations revealed that several $\mathrm{N}$-terminal residues of p15 may form salt-bridges and $\mathrm{H}$ bonds with CDK6 (magenta circle of top right panel with enlarged simulation in the bottom middle panel), while the p16/CDK6 complex lacks such interactions (bottom left and right panels show the minimum distance distributions of identified key interactions for p15/CDK6, compared with p16/CDK6, in MD simulations). Specifically, Arg24 of p15 may be a hotspot residue in the p15/CDK6 interactions, and Glu16 of p15 may form a salt bridge with Arg45, which will be stabilized in the position and form salt bridge with CDK6. b Sequence alignment of p15 and p16 using Clustal W 1.83 (ref. ${ }^{68}$ ). c, d Site-directed mutagenesis of the putative CDK6-binding residues in the N terminus p15 to alanine followed by inducible expression of Myc-tagged proteins in UMUC and immunoprecipitation (c) or by pull-down using E. coli-produced, histidinetagged mutant proteins in the presence of total proteins of UMUC cells (d). Note that the E16A, S20A, R24A, and R45A mutants had significantly reduced binding to both CDK4 and CDK6, although the mutational effects differed among the different mutants and binding detection methods (see "Results" for details). Experiments in $\mathbf{c}$ and $\mathbf{d}$ were repeated three times with similar results. Source data are provided as a Source Data file.

Using one-to-one residue mapping from sequence alignment of p15 and p16 (Fig. 6b), we found that Glu16, Ser20, Arg24, and Arg45 of p15 corresponded to Asp14, Thr18, Arg22, and Ser43 of p16, respectively. Structural and dynamics analysis of the p16-CDK6 complex indicates that, due to the repulsive force of Arg168 of CDK6 with Arg24 of p16 (corresponding to Leu26 of p15), the nearby Arg22 of p16 could not form salt-bridges with Glu18 and Glu21 of CDK6 (Fig. 6a). Our modeling therefore suggests a structural basis extending the experimental results indicating that p15 interacts more strongly with CDK6 than p16.

To experimentally validate our computational hypotheses, we carried out structurally guided site-directed mutagenesis by replacing Glu16, Ser20, Arg24, and Arg45 of p15 with alanine. Expression of these mutants fused with Myc-tag in UMUC3 followed by immunoprecipitation of CDK4 and CDK6 using antiMyc antibody or by pull-down experiment using histidine-tagged mutants as baits and total protein extracts from parental UMUC3 cells showed that, compared to wild-type p15, the p15 mutants had markedly reduced interactions with both CDK4 and CDK6 (Fig. 6c, d). While the extent of weakened interactions was somewhat dependent on different mutants, methods of detection, and the interactions with CDK4 versus CDK6, the overall results were highly consistent with our simulation results regarding the key amino acids that were suggested to be critical for the binding between p15 and CDK6 (Fig. 6a, b).

To further test whether the relatively weak interaction between p16 and CDK6 was due in part to the repulsive force between Arg24 of p16 (corresponding to Leu26 of p15) and Arg168 of CDK6, we 

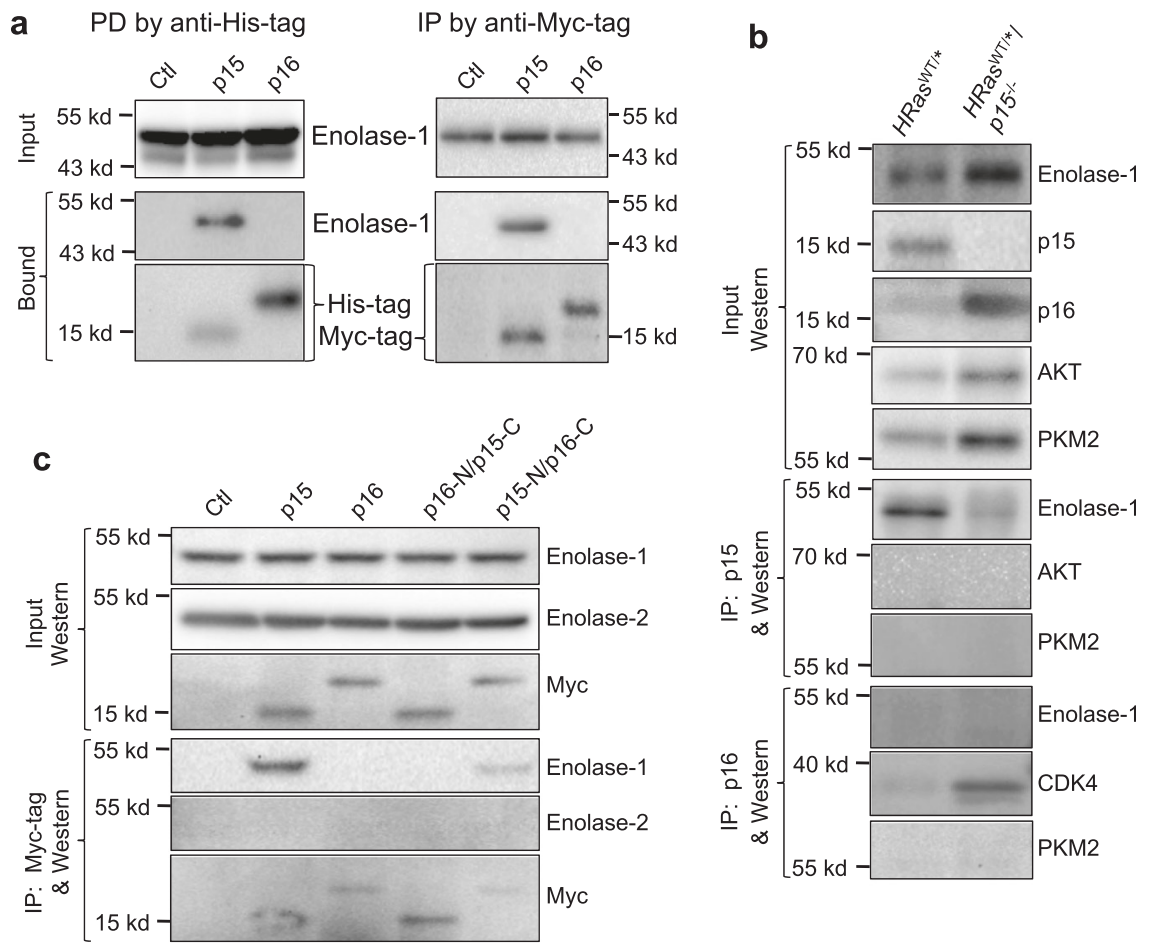

Fig. 7 p15, but not p16, binds enolase-1 in vitro and in vivo. a (left panel) Protein pull-down (PD) using E. coli-expressed, histidine-tagged and nickel column-immobilized p15 or p16 in the presence of total proteins from parental UMUC3 cells, and (a, right panel) immunoprecipitation (IP) of Myc-tagged p15 or p16, followed by western blotting. Note in both experiments the highly specific interaction of p15, but not p16, with enolase-1 with a molecular weight of $48-\mathrm{kDa}$. The side bars in the top two western blotting strips in the right panel denote molecular weight standards, 55-kDa (upper bar) and 43-kDa (lower bar). b p15 specifically interacts with enolase-1 in vivo. Total proteins were extracted from mouse urothelial cells expressing HRas ${ }^{\star}$ or those expressing HRas* and lacking p15, and were then subjected to immunoprecipitation followed by western blotting. One mouse per lane representative of a total of three independent mice per genotype. Note that enolase-1 was immunoprecipitated by anti-p15 antibody in p15-expressing, but not in p15-lacking urothelial cells (despite the fact that enolase-1 was significantly elevated in these cells); that p15 did not immune-precipitate AKT or PKM2; and that p16 did not immuno-precipitate enolase-1. c p15 binds, via its N terminus, to enolase-1. Myc-tagged, domain-switching mutants of p15 and p16 and wild-type controls were stably transfected and inducibly expressed in parental UMUC3 cells, and anti-Myc immunoprecipitation followed by western blotting was carried out. Note the specific immunoprecipitation of enolase-1, but not enolase-2, by p15 and p15-N/p16C, but not by p16 or p16-N/p15-C. Experiments in a-c were repeated three times with similar results. Source data are provided as a Source Data file.

replaced Arg24 of p16 with leucine, because MD simulations of p16-CDK6 complex showed that Arg22 of p16 $6^{\mathrm{R} 24 \mathrm{~L}}$ could partially recover the salt-bridges with Glu18 and Glu21 of CDK6 (Supplementary Fig. 6a and 6b). Such a replacement indeed increased the binding between $\mathrm{p} 16$ and CDK6, but not with CDK4 (Supplementary Fig. 6c), suggesting that other residues in p16 that are divergent from p15 may also be less optimal for interacting with CDK4.

p15 Interacts via its $N$ terminus with enolase- 1 in vitro and in vivo. Having established that $\mathrm{p} 15$ is a more potent cell-cycle inhibitor than p16, we explored whether p15 played a non-canonical role in tumor suppression through interaction with other cellular protein(s). We took an unbiased pull-down approach using E. coliproduced, histidine-tagged p15 as a bait, with $E$. coli-produced histidine-tagged p16 or histidine-tag only as controls. After coupling the bait and controls separately onto nickel columns, we incubated them with total proteins extracted from parental UMUC3 cells. The bound proteins were eluted and resolved by two-dimensional electrophoresis, after which the proteins specifically bound to histidine$\mathrm{p} 15$, but not to histidine alone or to histidine-p16, were selected for mass spectrometry (Supplementary Fig. 7). Of the 17 two-D gel spots analyzed, two belonged to enolase-1 (alpha enolase), one receiving a mascot score of 1823 with 117 peptide sequences identified by liquid chromatography tandem mass spectrometry (Supplementary Table 1).
To determine whether the interaction between p15 and enolase1 was specific, we performed pull-down using E. coli-produced, histidine-tagged $\mathrm{p} 15$ and total proteins from parental UMUC3, as well as immunoprecipitation using inducible expression of Myctagged p15 in UMUC3 cells. Both experiments unequivocally validated the specific binding between p15 and enolase-1 (Fig. 7a). In contrast, under identical experimental conditions, p16 failed to bind enolase-1. p15, but not p16, also bound to enolase-1 in vivo in mouse urothelial cells expressing oncogenic HRas (Fig. 7b). Notably, p15-lacking/HRas-expressing urothelial cells had significantly elevated enolase-1 (Fig. 7b), which is consistent with upregulated glycolysis in tumor cells (see later). Additionally, whereas p15 bound to enolase-1, it did not bind to AKT or pyruvate kinase 2 (PKM2) (Fig. 7b), thus further demonstrating the binding specificity. Utilizing the aforementioned p15/p16 domain-switching mutants (as shown in Fig. 5), we further showed in enforced expression and immunoprecipitation experiments that it was the $\mathrm{N}$ terminus of p15 that mediated the interaction between p15 and enolase-1 (Fig. 7c). Finally, neither wild-type p15 nor p15-N/p16-C hybrid bound to enolase-2 (neuron-specific enolase) (Fig. 7c).

Competitive binding among p15, enolase- 1 and CDK4 and its effects on enolase-1 activity and aerobic glycolysis. To assess whether the binding between p15 and enolase-1 reduces the 


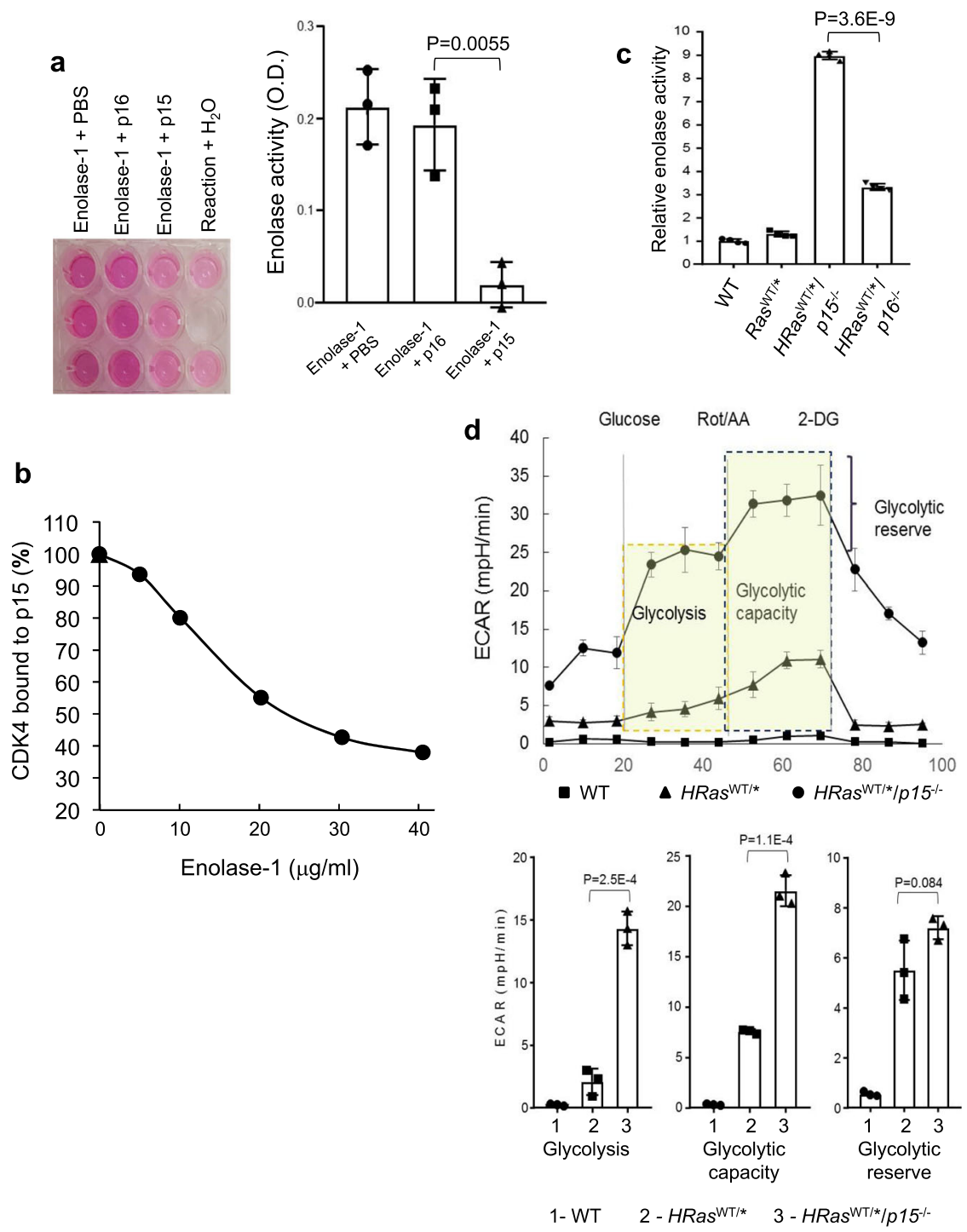

Fig. 8 The competitive binding between p15, enolase-1, and CDK4/CDK6. a p15, but not p16, inhibits enolase-1 activity in vitro. Purified enolase-1 was mixed with PBS, E. coli-produced p15 or E. coli-produced p16, and the enolase activity was measured using a commercial kit and expressed as relative optical density (OD). Note that $p 15$, but not p16, inhibits enolase-1 activity. $n=3$ of biologically independent samples. Data are presented as mean values \pm SD. Two-sided $t$-test was performed to compare the significance of the difference between the two groups (p15 versus p16). The $P$ values are shown in the figure. b Enolase-1 competitively inhibits p15 from binding to CDK4 in a dose-dependent manner. Purified enolase- 1 was mixed with E. coli-produced p15, and the mixture was incubated with immobilized CDK4. Note that enolase-1 in a dose-dependent manner competitively inhibits p15 from binding to CDK4. $n=3$ of biologically independent samples. Data are presented as mean values \pm SD. c Enolase activity in relation to p15 and p16 status in vivo. Total

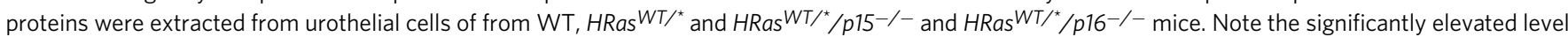
of enolase activity in urothelial cells lacking p15. $n=4$ of biologically independent samples. Data are presented as mean values \pm SD. Two-sided $t$-test was

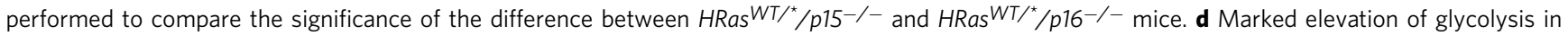
compound mice expressing HRas ${ }^{\star}$ and lacking p15. Seahorse analysis was performed using urothelial cells from WT, HRas WT/ ${ }^{*}$, and $H R a s^{W T /{ }^{*} / p 15^{-} /-}$mice. $n=3$ of biologically independent samples. Data are presented as mean values \pm SD. Two-sided $t$-test was performed to compare the significance of the difference between the two groups (HRas $\mathrm{WT}^{*} / \mathrm{p}^{1} 5^{-/-}$versus HRas ${ }^{\mathrm{WT}} /{ }^{*}$ ). The $P$ values are shown in the figure. Note that glycolysis, glycolytic capacity, and glycolytic reserve were all significantly elevated in $H R a S^{W T /{ }^{*}} / \mathrm{p} 15^{-/-}$than in the controls (see lower panel for quantified comparisons).

catalytic activity of latter, we incubated the purified proteins in vitro, and found that $\mathrm{p} 15$, but not $\mathrm{p} 16$, significantly reduced the activity of enolase-1 (Fig. $8 \mathrm{a}$ ). Because the $\mathrm{N}$ terminus of p15 mediates its binding to both CDK4 and enolase-1, we next examined whether the binding of enolase-1 to p15 could competitively inhibit CDK4 from binding to p15. In a plate assay, with increasing concentrations of enolase-1, we observed a dose-dependent decrease of the binding of CDK to p15 (Fig. 8b).
Consistent with the cell-free system, we found a strong correlation between p15 deficiency and elevated enolase activity in vivo. For instance, the enolase activity was about threefold higher in HRas WT/ */p15 $15^{-1-}$ mice than in $H R a \mathrm{STT}^{*} / \mathrm{p} 16^{-1-}$ mice (Fig. 8c). Furthermore, by Seahorse analysis, the glycolysis, glycolytic capacity, and glycolytic reserve were all markedly elevated in $\mathrm{HRas} \mathrm{WT}^{*} / \mathrm{p} 15^{-1-}$ mice that lacked p15 expression (Fig. 8d). Together, our data reveal a role of p15 in regulating tumor cell metabolism via its interaction and inhibition of enolase-1 and glycolytic activities. 

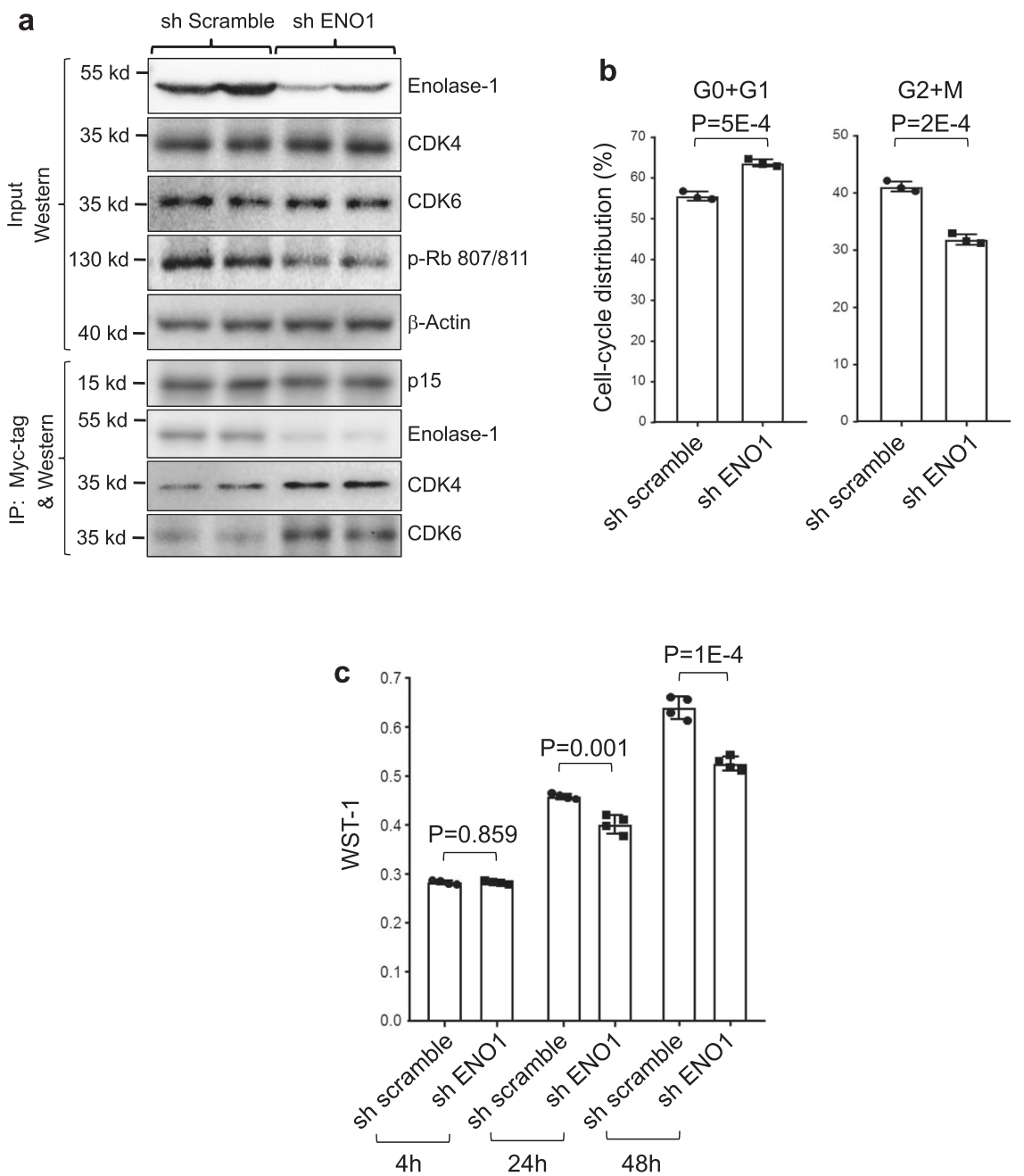

Fig. 9 Down-regulation of enolase-1 reduces bladder cancer cell proliferation. a knockdown of enolase-1 by shRNA in UMUC3 cells leads to increased binding of p15 to CDK4 and CDK6 as evidenced by immunoprecipitation followed by western blotting. The two lanes represented two stable clones from each of scrambled control and enolase-1 shRNA. Source data are provided as a Source Data file. b, c Reduced enolase-1 was associated with reduced cell-cycle progression and increased cell proliferation. $n=3$ and 4 for $\mathbf{b}$ and $\mathbf{c}$, respectively, of biologically independent samples. Data are presented as mean values \pm SD. Two-sided $t$-test was performed to compare the significance of the difference between the two groups ( $p 15$ versus p16). Experiments were repeated three times with similar results.

Down-regulation of enolase-1 suppresses bladder cancer cell proliferation. To begin to address the biological effects of enolase-1, we down-regulated its expression using shRNA knockdown in UMUC cells expressing p15 (Fig. 9a). Compared to the shamble controls, the reduction of enolase- 1 was associated with an increased binding between p15 and CDK4 and CDK6 (Fig. 9a) and reduced cell-cycle progression (Fig. 9b) and cell proliferation (Fig. 9c), thus lending further support to competitive nature observed in vitro of the binding among p15, CDK4/6, and enolase-1. These data further suggest that p15 plays a dual role in suppressing cell-cycle progression and glycolysis.

The findings we presented in this paper are highly significant on several fronts. First, the multiple lines of evidence we obtained in vitro and in vivo establish $C D K N 2 B$ as a central player, rather than a bystander, of $9 \mathrm{p} 21.3$ locus in urothelial tumor suppression and the dominant role of $C D K N 2 B$ deficiency in driving the formation of low-grade non-invasive bladder cancer. In doing so, we resolve a major discrepancy between the high prevalence of 9p21.3 deletion ( $70 \%)$ along with RTK-Ras activation $(\sim 95 \%)$ in human bladder tumors $7,31,41$ and the lack of tumorigenicity in mice by activated RTK-Ras pathway components alone or in combination with CDKN2A deletion 19,42,43. Our data strongly suggest that
$C D K N 2 B$ deficiency is more reflective of $9 \mathrm{p} 21.3$ loss and is a functionally relevant collaborative partner with RTK-Ras activation to initiate bladder tumors. Consequently, the status of $C D K N 2 B$, instead of CDKN2A, coupled with RTK-Ras activation should be used as a combinatorial biomarker set to diagnose and predict the recurrence of low-grade non-invasive bladder cancer. Because concurrent 9p21.3 inactivation and RTK-Ras activation are highly prevalent in non-bladder cancer types $8,9,13$, our findings call attention to the need to reexamine the effects of CDKN2B deficiency on tumorigenesis of other organ systems and cell types. Even greater attention should be paid to the tumorigenic role of $C D K N 2 B$ deficiency in tumors where $C D K N 2 B$ is preferentially deleted, such as familial melanoma, glioma, and certain hematological malignancies $2,12,44,45$. Second, our data reveal CDKN2B as an important player that interconnects cell cycle and glycolysis by specifically binding and inhibiting both CDK4/CDK6 and enolase1. Thus, the deficiency of $C D K N 2 B$ not only promotes tumor cell proliferation by releasing the constraints of $C D K N 2 B$ on $\mathrm{G} 1$ to $\mathrm{S}$ progression, it also creates a metabolically conducive environment during tumor-induced hypoxia under which tumor cells require increased glycolysis to produce metabolic intermediates for biosynthesis of cell building blocks, such as amino acids, lipids, 
and nucleic acids ${ }^{46}$. By compiling gene expression data (Metabolic gEne RApid Visualizer (http://merav.wi.mit.edu/) comprising eight normal bladder cohorts and nine bladder tumor cohorts from human patients (https://www.cbioportal.org/), we found enolase-1 to be significantly overexpressed in all the tumor cohorts irrespective of tumor grades, compared to the normal controls (Supplementary Fig. 8). These data are consistent with two earlier microarray-based gene expression cohorts that had information on tumor stages, showing that enolase- 1 is considerably higher in TaT1 non-muscle-invasive bladder tumors as well as in T2-T4 muscleinvasive tumors than in normal bladder specimens (Supplementary Fig. 9) ${ }^{47,48}$. It is worth noting that, within the muscle-invasive tumors, enolase-1 is generally the only overexpressed enolase isoform, instead of enolase-2 and enolase-3 (Supplementary Fig. 9). This is in agreement with the fact that normally enolase- 2 and enolase- 3 are primarily expressed in neurons and muscle cells, respectively, and are probably not used as the principal enolases for metabolism by normal urothelial cells. The specific upregulation and the unopposed activity of enolase- 1 in the absence of $C D K N 2 B$ in bladder tumor cells should open an avenue for therapeutic inhibition. Given the possibility that, even when $C D K N 2 B$ is wildtype, enolase-1 could competitively inhibit it from binding to CDK4 and CDK6 (Figs. 8 and 9), blocking enolase-1 could potentially release $C D K N 2 B$ and restore its activity as a cell-cycle inhibitor. Reprogramming the way by which glucose is utilized in bladder tumor cells by shifting glycolysis to oxidative phosphorylation may help stave off hypoxia-induced tumor cell proliferation and reduce the growth of low-grade superficial papillary bladder tumor growth and/or prevent it from recurring. The feasibility to deliver chemical agents directly into the bladder via a trans-urethral catheter should help attain the highest possible local concentration with lowest possible systemic toxicity ${ }^{49}$. Finally, our data should have implications beyond bladder cancer and cancers in general, because 9 p21.3 is the most prominent susceptibility locus that is strongly associated with patients with coronary artery disease $\mathrm{e}^{21,50,51}$ and with type 2 diabetes 22,23 . While these sequence variants, i.e., rs10757278-G for coronary artery disease and rs10811661-T for type 2 diabetes, are located outside the coding region of $C D N K 2 B$, they have been shown to affect the activity of ANRIL, which in turn could downregulate $C D N K 2 B$ expression ${ }^{52}$. Data from our present study regarding $C D K N 2 B$ as a potent dual inhibitor of cell cycle and glycolysis should open new avenues to establish the disease linkage on a functional level and elucidate the cellular mechanisms whereby 9 p21.3 aberrations lead to coronary artery disease, type 2 diabetes, and glaucoma.

\footnotetext{
Methods

Genetically engineered mice. Upk2-HRas is a transgenic mouse line originally developed in the corresponding author's laboratory in which a $3.6-\mathrm{kB}$ murine uroplakin II promoter drives urothelium-restricted expression of a constitutively active rabbit HRas oncogene (Q61L) ${ }^{27}$. Heterozygous Upk2-HRas which has been successfully maintained to date harbors a single-copy Upk2-HRas transgene and reproducibly develops simple urothelial hyperplasia of the bladder ${ }^{19}$. This line serves as breeders and a reference line representing tumor precursor lesion of the urothelium. CDKN2B (p15) knockout mouse line (a kind gift of Dr. Angel Pellicer of New York University School of Medicine) is a ubiquitous p15 knockout line in which the exon 2 of p15 gene was replaced with a neo, which abrogated p15 expression $^{53}$. p16 knockout mouse line, obtained from US National Cancer Institute's Mouse Repository, is a ubiquitous p16 knockout line in which the exon $1 a$ of CDKN2A gene was replaced with a neo gene, which inactivated p16 but not alternatively transcribed p19 (ref. ${ }^{33}$ ). Ink4ab (e.g., p15/p16) double knockout mouse line (a kind gift of Dr. Anton Berns of The Netherlands Cancer Institute) is a ubiquitous knockout line that was originally obtained in Dr. Berns's laboratory by targeting p15 in the p16 knockout ES cells where exon 2 of CDKN2A bore a stop codon in the p16 reading frame but not in the p19 reading frame ${ }^{18}$. Together, this abrogated the expression of p15 and p16 without affecting the p19 expression. Intercrosses were carried out between the heterozygous Upk2-HRas line and each of aforementioned knockout (KO) lines, and the offspring was further intercrossed such that the Upk2-HRas transgene was heterozygous and the $\mathrm{KO}$ alleles were hetero- or homozygous for tumorigenic analyses. The Upk2-HRas transgene allele
}

was identified by genomic Southern blotting of NcoI-digested tail DNA or by genomic PCR using specific primers (Supplementary Table 2). Knockout alleles and wild-type control alleles for p15, p16, and Ink4ab (p15/p16) were also verified by genomic PCR using specific primers (Supplementary Table 2).

All animal-related procedures were carried out in accordance with local and federal regulations and after the approval of a protocol by Institutional Animal Care and Use Committee (IACUC) of New York University School of Medicine.

\section{Urothelial tumorigenesis analyses}

Urine dipstick test. Preliminary screening of hematuria, a common manifestation of bladder tumors in human patients, was performed on spot urine samples collected from conscious mice through gentle massaging of the lower abdomen. Urine samples were immediately applied atop of dipstick strips (BD Biosciences). The color change from yellow to blue on the sixth square from the top was considered positive for hematuria and the result was recorded on a digital camera.

Gross anatomy and histopathology. Mice of unique genotypes comprising different cohorts were sacrificed at pre-determined time points, and their urinary bladders were freshly dissected out, urine drained, weighed, turned inside out for the presence of visible tumors. The bladders were then bisected along the sagittal plane, with one half fixed in $10 \%$ PBS-buffered formalin, routinely dehydrated in serial ethanol and xylene, embedded in paraffin, sectioned, and stained with hematoxylin and eosin, and the other half scraped off its mucosa/tumors for protein preparation and biochemical work.

Bladder cancer cell lines, stable transfection, and inducible gene expression. Two human bladder cancer cell lines, RT112 and UMUC3, that had 9p21.3 deletion $^{37}$ were purchased from the American Type Culture Collection (Manassas, VA, USA) and used for all transfection experiments. Both the cell lines were cultured in Dulbecco's modified Eagle's medium (DMEM) (Gibco, Grand Island, NY, USA) supplemented with $10 \%$ Tet-system approved fetal bovine serum (Clontech, Mountain View, CA, USA) at $37^{\circ} \mathrm{C}$ with $5 \% \mathrm{CO}_{2}$.

A tetracycline-based inducible system was chosen for conditional gene expression. Briefly, plasmid pTet-on (Clontech, Mountain View, CA, USA) was transfected into RT112 and UMUC3 cells using Lipofectamine 2000 (Invitrogen, Carlsbad, CA, USA), followed by selection of stable clones in $300 \mu \mathrm{g} / \mathrm{ml} \mathrm{G418}$ (Gibco, Grand Island, NY, USA). pTRE2-pur containing p15, p16, p15-N/p16-C or p16-N/p15-C was then transfected into pTet-on-stably transfected RT112 or UMUC3 using Lipofectamine 2000 and stable clones were selected in the presence of puromycin (Gibco) $(0.8 \mu \mathrm{g} / \mathrm{ml}$ for RT112 and $1.2 \mu \mathrm{g} / \mathrm{ml}$ for UMUC3).

\section{Cell cycle and proliferation analyses}

Proliferation assay. Cultured bladder cancer cells $\left(5 \times 10^{3}\right)$ were seeded onto 96 well plates in DMEM medium with FBS containing $1 \mu \mathrm{g} / \mathrm{ml}$ doxycycline, and cell proliferation was determined at 24,48 , and $72 \mathrm{~h}$ by WST-1 (Clontech, Mountain View, CA, USA) with a 96-well plate reader at $450 \mathrm{~nm}$ absorbance (Dynex, Chantilly, VA, USA).

Fluorescence-activated cell sorting. Cultured cells were detached by trypsin digestion, collected, and washed by centrifugation at $800 \times g$ for $5 \mathrm{~min}$. The cells were then fixed in pre-cooled, $70 \%$ ethanol, washed in PBS, and stained with propidium iodide/RNase staining solution (Cell Signaling Technology, Danvers, MA, USA) and sorted with a FACSCalibur analyzer, and the data were analyzed by FlowJo software (FlowJo 10.4.2).

E2F activity assay. E2F activity was assessed by transient transfection of UMUC3 cells with an E2F-luciferase reporter construct (Cignal E2F Reporter Assay Kit, Qiagen). Briefly, UMUC3 cells that reached $60 \%$ confluence were transfected the E2F reporter plasmid in the presence of Lipofectamine 2000 (Invitrogen, carlsbad, CA, USA). After $12 \mathrm{~h}$ of incubation, the transfected cells were lysed, supplemented with dual-luciferase reporter assay kit (Promega), and the luciferase activity was measured using a luminometer (Synergy H4 Hybrid Reader, Biotek,Winooski, VT, USA).

\section{Computational and MD simulation of p15-CDK6 interactions}

Computational modeling. Because the crystal structure of p15 is unavailable, the structure of p16-CDK6 complex (PDB id: 1 bi7) ${ }^{40}$ was used as a template to model the molecular interactions between p15 and CDK6. The existing p16-CDK6 model missed two structural parts: one in CDK6 (residues 49-71) and the other in the N terminus of p16 (residues 1-9) (Supplementary Fig. 5a). The Modeler Program ${ }^{54}$ was used to model these two missing parts. For the non-terminal missing part of CDK6, a highly similar CDK6 structure from the p18-CDK6-K-cyclin complex (PDB: $1 \mathrm{~g} 3 \mathrm{n})^{55}$ was used as the template to build the missing part (Supplementary Fig. 5b). For the missing part of the $\mathrm{N}$ terminus of p16, no template was used in the modeling procedure. For the p15-CDK6 complex, protein modeling of p15 (sequence 1-138) was performed on the web server of I-TASSER ${ }^{56,57}$ following the standard instructions. The identified structural templates from the PDB database were p16 from the p16-CDK6 complex (PDB id: 1 bi7) ${ }^{40}$ and p15 from p15 NMR 
ensemble (PDB id: $1 \mathrm{~d} 9 \mathrm{~s})^{58}$. Among the five p15 models predicted by I-TASSER based on p16, the one with the highest $C$-score was selected and superimposed on the p16-CDK6 complex to build p15-CDK6 complex (Supplementary Fig. 5d).

MD simulation. Two comparable systems: $\mathrm{p} 16-\mathrm{CDK} 6$ and $\mathrm{p} 15-\mathrm{CDK} 6$ complexes as well as an additional p16(R24L)-CDK6 complex were investigated in this study. For each system, five independent MD replicas were carried out using same starting structural configuration, but with different initial velocities. Total simulation time of 2.25 s was performed (Supplementary Table 3 ). All MD simulations were carried out with Amber 16 package using Amber FF 14SB force field ${ }^{59,60}$. AmberTools ${ }^{61}$ were used to create topology and coordinate files for the structures. Each system was neutralized by adding counterions of $\mathrm{Na}^{+}$or $\mathrm{Cl}^{-}$, and was solvated in rectangular water box full of TIP3P water, so that the boundary of the box is at least $12 \AA$ away from any solute atom. The cutoff distance was set at $10 \AA$ for short-range Coulomb and van der Waals interactions, and the particle mesh Eward (PME) method ${ }^{62}$ was utilized to treat long-range electrostatic interactions. The SHAKE algorithm ${ }^{63}$ was employed on all atoms covalently bonded to hydrogen atom, allowing for an integration time step of $2 \mathrm{fs}$. The simulation detail is as follows. Each system was minimized by three steps: (i) the system was minimized by 3000 steps of the steepest descent minimization followed by up to 2000 steps of conjugate minimization, and harmonic restraints with a force constant of $5 \mathrm{kcal} / \mathrm{mol} \AA$ were applied to all solute atoms; (ii) the above minimization procedure was repeated and harmonic restraint with a force constant of $1 \mathrm{kcal} / \mathrm{mol} \AA$ imposed on solute atoms; and (iii) no harmonic restraint was imposed on solute atoms during the minimization. After minimization, each run was subjected to equilibration in following steps: (i) $50 \mathrm{ps}$ constant volume ensemble (NVT) MD simulations with $2 \mathrm{kcal} / \mathrm{mol} \AA$ restraints on the solute atoms, and the system was gradually heated from 10 to $300 \mathrm{~K}$. (ii) $50 \mathrm{ps}$ NVT MD at $300 \mathrm{~K}$ and with $2 \mathrm{kcal} / \mathrm{mol} \AA$ restraints on the solute atoms. (iii) 100 ps isothermal isobaric ensemble (NPT) MD at $300 \mathrm{~K}$ and with $0.5 \mathrm{kcal} / \mathrm{mol} \AA$ restraints on the solute to adjust the solvent density. Finally, production MD simulations were carried out for $150 \mathrm{~ns}$ at a constant temperature of $300 \mathrm{~K}$ and a constant pressure of $1 \mathrm{~atm}$. The temperature and pressure were maintained using Langevin thermostat and Berendsen barostat methods, respectively. The atomic coordinates of the complexes were saved every $100 \mathrm{ps}$ to obtain the trajectories for post structural analysis.

Post structural analysis of MD trajectory. The root-mean-square deviation (RMSD) profiles of protein $\mathrm{C}$ alpha atoms (Supplementary Fig. 10a) and $B$-factor analysis (Supplementary Fig. 10b) for p15-CDK6, p16-CDK6, and p16 R24L $_{-} \mathrm{CDK} 6$ complexes in different MD replicas were performed. For each MD replica, the residue Bfactor analysis was conducted on the last 50-ns trajectories using atomicfluct command in cpptraj module of AmberTools ${ }^{61}$. The residue $B$-factor is the massweighted average of positional fluctuations squared of all atoms in each residue multiplied by a constant $8 \pi^{2} / 3$. For each system, a combined 500 -ns trajectories $(5$ MD replicas of last 100-ns trajectories) were aligned and clustered. The clustering was performed on the binding interface residues by using the DBSCAN method ${ }^{64}$ with min-points of 50 and epsilon of $1.2 \AA$, which is implemented in cpptraj module of AmberTools ${ }^{61}$. For the p15-CDK6 system, nine clusters were generated and the most populated one (832 out of 5000 snapshots) was selected for p15-CDK6binding interface analysis (Fig. 6a). Besides the clustering analysis, the minimum distance distributions of identified key interactions were carried out over combined 500-ns trajectories for p15-CDK6, compared with corresponding residue pairs in p16-CDK6 (Fig. 6a), as well as those in p16 ${ }^{\mathrm{R} 24 \mathrm{~L}}$-CDK6 (Supplementary Fig. 6b).

\section{Construction of expression vectors containing wild-type cDNA, hybrids, deletion, and point mutations}

Myc-tagged $p 15$ and $p 16$. To facilitate detection and minimize artifacts with the use of different antibodies for immunoprecipitation and western blot-based semiquantification, a Myc-tag was inserted in-frame at the C terminus of wild-type p15 and p16 cDNAs (Addgene, Watertown, MA) by PCR using Q5 High-Fidelity DNA Polymerase (New England BioLabs), with p15- and p16-specific primers (Supplementary Table 2). The PCR products were then purified, sequence-verified, and cloned into pTRE2pur vector (Clontech) for transfection experiments.

p15/p16 domain-switch constructs. Two different hybrids, one comprising the $\mathrm{N}$ terminus of p15 and the C terminus of p16 (p15-N/p16-C) (1-methionine to 51-isoleucine of p15/52-glutamine to 156-aspartate of p16) and another comprising the $\mathrm{N}$ terminus of $\mathrm{p} 16$ and the $\mathrm{C}$ terminus of $\mathrm{p} 15$ (p16-N/p15-C) (1-methionine to 49-isoleucine of p16/51-isoleucine to 138-aspartate of p15) were constructed using the following procedures. First, PCR was carried out using pTRE2pur-p15-Myc as a template using primer 1 (see Supplementary Table 2 as with all other primers) and primer 2. Second, PCR was carried out using pTRE2pur-p16-Myc as a template, using primer 3 and primer 4 . Third, the products from the above two steps were mixed and run for 10 PCR cycles, and then primer 1 and primer 4 were added and 30 additional PCR cycles were run. These steps yielded p15-N/p16-C. To generate p16-N/p15-C, pTRE2pur-p16-Myc was used as a template for PCR using primer 5 and primer 6. This was followed by PCR using pTRE2pur-p15-Myc as a template using primer 7 and primer 4 . The PCR products from the above two steps were mixed and run for 10 PCR cycles. Primer 5 and primer 4 were then added and 30 additional PCR cycles were carried out. These steps yielded p16-N/p15-C. The p15-
N/p16-C and p16-N/p15-C cDNA hybrids were cloned separately into pTRE2pur vector for transfection experiments.

Construction of histidine-tagged $p 15$ and $p 16$ and recombinant protein production in E. coli. Histidine (His)-tagged p15 and -p16 were amplified using pTRE2purp15-Myc and pTRE2pur-p16-Myc as templates, respectively, by PCR using Q5 High-Fidelity DNA Polymerase (New England BioLabs). The primers for Histagged p15 and His-tagged p16 are listed in Supplementary Table 2. The PCR products were cloned into pET28a (Millipore) and transformed to into BL21(DE3) competent E. coli (New England BioLabs) to produce recombinant His-tagged p15 and His-tagged p16 after the induction by IPTG. The His-tagged proteins were purified on Ni-NTA Superflow Columns (Qiagen).

p15 N-terminal deletion mutants. The $\mathrm{N}$-terminal deletion fragments of p15 cDNA were generated by PCR using wild-type p15 cDNA as template with Q5 SiteDirected Mutagenesis Kit (New England BioLabs), with specific primers for $\Delta 2-15$, $\Delta 2-27, \Delta 2-37$, and $\Delta 2-49$ as listed in Supplementary Table 2.

Point mutation of p15. Point mutations were created using site-directed mutagenesis in p15 using two sets of plasmids as templates, i.e., pTRE2pur-p15-myc-tag and pET28a-p15-his-tag, which were used for bladder cancer cell line transfection and $E$. coli transformation, respectively. The mutagenesis primers for $\mathrm{p} 15 / \mathrm{E} 16 \mathrm{~A}$, p15/S20A, p15/R24A, and p15/R45A are listed in Supplementary Table 2.

\section{Protein-protein interaction and semi-quantification}

Co-immunoprecipitation. Cultured UMUC3 bladder cancer cells were lysed in RIPA buffer ( $25 \mathrm{mM}$ Tris-HCl, pH 7.6, $150 \mathrm{mM} \mathrm{NaCl}, 1 \%$ [v/v] NP-40). The soluble proteins were incubated with mouse anti-Myc-tag (Supplementary Table 4). After gentle rocking at $4^{\circ} \mathrm{C}$ overnight, agarose-protein A/G beads (sc-2003, Santa Cruz Biotechnology, USA) were then added and incubated at $4{ }^{\circ} \mathrm{C}$ for $4 \mathrm{~h}$. The beads were collected by centrifugation, washed, and boiled for $5 \mathrm{~min}$ in SDS-PAGE loading buffer. The immunoprecipitated proteins were detected by western blotting using specific antibodies (Supplementary Table 4).

Pull-down. Total protein lysates were prepared from cultured UMUC3 cells in RIPA buffer and then incubated with His-tagged proteins produced in E. coli/BL21 with gentle rotation at $4{ }^{\circ} \mathrm{C}$ overnight. The mixture was loaded onto Ni-NTA Superflow Columns (Qiagen), incubated and washed, and the bound proteins were eluted with different concentrations of imidazole solution. Western blotting was performed to detect the proteins in each eluted fraction. The bound proteins on western blotting from both immunoprecipitation and pull-down experiments were semi-quantified by scanning the protein bands using NIH ImageJ software.

Competitive inhibition of CDK binding to p15 by enolase-1. p15 protein purchased from Abcam was dissolved in $100 \mu \mathrm{l}(2.5 \mu \mathrm{g} / \mathrm{ml})$ coating buffer (Bio-Rad, Kidlington, UK) and pre-coated onto a clear flat-bottom 96-well plate (Thermo Fisher Scientific) at $4{ }^{\circ} \mathrm{C}$ overnight. After washing three times with $\mathrm{PBS}$, the pre-coated wells were blocked with $1 \%$ bovine serum albumin (BSA) (W/V) in PBS at $37^{\circ} \mathrm{C}$ for $1 \mathrm{~h}$, after which the wells were washed three times with PBS and supplemented with CDK4 $(5 \mu \mathrm{g} / \mathrm{ml}$ in 100 $\mu \mathrm{l}$ ) (Novus) in the presence of $0-40 \mu \mathrm{g} / \mathrm{ml}$ of enolase-1 (Sino Biological Inc., Wayne, PA). Following additional incubation at $37^{\circ} \mathrm{C}$ for $2 \mathrm{~h}$, the wells were washed in PBS containing $0.05 \% \mathrm{v} / \mathrm{v}$ Tween-20 and incubated with $100 \mu \mathrm{l}$ rabbit anti-CDK4 antibody (Supplementary Table 4 ) at $37^{\circ} \mathrm{C}$ for $1 \mathrm{~h}$. After washing three times in PBS, $100 \mu \mathrm{l}$ HRP-conjugated goat anti-rabbit IgG antibody (Supplementary Table 4) was added, incubated, and developed in $100 \mu \mathrm{l}$ of the TMB substrate solution (Thermo Fisher Scientific). Absorbance was read in a microplate reader (Dynex) immediately at the 450 $\mathrm{nm}$ after adding $100 \mu \mathrm{l}$ stop solution (Thermo Fisher Scientific).

Changes in p15 binding to CDK4 and CDK6 after enolase-1 knockdown. A Lentiviral vector (Origene) was reengineered to bear an shRNA of enolase-1 (GCATTGGAGCAGAGGTTTACC-TCAAGAG (loop)-GGCGTTCAATGTCAT CAATGG). The lentivirus was packaged in $293 \mathrm{~T}$ cells by co-transfection with the plasmids, VSV-G, PAX2, and the shRNA-bearing vector. The packaged lentivirus was then used to transfect UMUC3 cells harboring Myc-tagged p15, and the stable transfected cells were selected in the presence of $4 \mu \mathrm{g} / \mathrm{ml}$ of blasticidin (InvivoGen). Co-immunoprecipitation of p15 with CDK4 and CDK6 as well as cell cycle and cell proliferation analyses were described above.

\section{Two-dimensional gel electrophoresis and mass spectrometry of p15-bound} proteins. Recombinant proteins (p15-Myc, p16-Myc, and control (myc-tag only)) produced in E. coli that served as baits were separately immobilized on Ni-NTA Superflow Columns and incubated with total proteins extracted from parental UMUC3. After washing, the bound proteins were eluted with imidazole. Two-dimensional electrophoresis was performed based on the carrier ampholyte method of isoelectric focusing ${ }^{65,66}$. Briefly, the first dimension (e.g., isoelectric focusing) was performed using 2\% pH 3-10 Isodalt Servalytes (Serva, Heidelberg, Germany). Tropomyosin (MW 33,000 and pI 5.2) served as an internal standard. After equilibration in $10 \%$ glycerol, $50 \mathrm{mM}$ dithiothreitol, $2.3 \% \mathrm{SDS}$, and $0.0625 \mathrm{M}$ tris, $\mathrm{pH} 6.8$, the first-dimensional gel 
was loaded atop a 10\% SDS-PAGE, with molecular weight standards (Sigma Chemical Co., St. Louis, MO and EMD Millipore, Billerica MA): myosin $(220,000)$, phosphorylase A $(94,000)$, catalase $(60,000)$, actin $(43,000)$, carbonic anhydrase $(29,000)$, and lysozyme $(14,000)$. Protein spots that were not present in the control or p16-bound fractions were excised, in-gel trypsin-digested, HPLC-purified and subjected to mass spectrometry following established procedures ${ }^{67}$.

\section{Glycolysis analysis}

Enolase activity assay. Cultured bladder cancer cells $\left(2 \times 10^{6}\right)$ were lysed with icecold Enolase Assay Buffer (Biovision, Milpitas, CA, USA). The enolase activity and standard curve were both determined according to the instructions of enolase activity colorimetric assay kit (Biovision).

Seahorse analysis. Urothelial cells were harvested from the mucosal surfaces of the inside-out mouse bladders. The cells were incubated in a Liberase solution at $37^{\circ} \mathrm{C}$ for $15 \mathrm{~min}$, after which the digestion was stopped by adding $2 \%$ fetal bovine serum and $0.2 \mu \mathrm{M}$ EDTA. The cells were then cultured in cell culture microplates precoated with poly-L-lysine (Seahorse Bioscience, Santa Clara, CA, USA) at $3 \times 10^{4}$ cells/well density in $500 \mu \mathrm{l} \mathrm{XF}$ Base DMEM medium (Agilent, Santa Clara, CA, USA) and incubated at $37^{\circ} \mathrm{C}$ in $5 \% \mathrm{CO}_{2}$. The assays were conducted at different time points by adding glucose, Rot/AA, and 2-DG successively in an XFe24 extracellular flux analyzer (Agilent, Santa Clara, CA, USA), and the ECAR data were recorded automatically.

Immunofluorescence staining and western blotting. For staining the deparaffinized sections were subjected to antigen retrieval by microwaving at the maximum output in antigen unmasking solution (Vector Laboratories, Burlingame, CA, USA) for $20 \mathrm{~min}$. After incubation in $4 \%$ Triton X-100 in PBS, the non-specific sites were blocked with $1 \%$ BSA in PBS. The primary antibodies used for immunofluorescence staining and their dilutions are listed in Supplementary Table 4. After incubation with primary antibody at $4{ }^{\circ} \mathrm{C}$ overnight, the sections were washed with PBS three times, and then incubated with Alexa-488-conjugated goat anti-mouse or Alexa594-conjugated goat anti-rabbit secondary antibody (Invitrogen; $1: 500$ dilution) at $37^{\circ} \mathrm{C}$ for $1 \mathrm{~h}$. After nuclear counterstaining with $1 \mu \mathrm{g} / \mathrm{ml}$ DAPI, the sections were mounted with aqua-mount (Lerner laboratories, Kalamazoo, MI, USA).

For western blotting, cultured human bladder cancer cells or mouse tissues were lysed in whole-cell lysis buffer containing $20 \mathrm{mM}$ Tris, pH 7.6, $50 \mathrm{mM} \mathrm{NaCl}, 10 \% \mathrm{SDS}$, protease, and phosphatase Inhibitors (Thermo Scientific, Rockford, IL, USA). The protein concentrations were determined by Pierce BCA Protein Assay Kit (Thermo Scientific), and $30 \mu \mathrm{g}$ of the proteins each sample were resolved on SDS-PAGE. The proteins were then electrophoretically transferred onto PVDF membrane, blocked with 5\% BSA in TBST, and incubated with the primary antibodies with proper dilutions (Supplementary Table 4). The secondary antibodies used were: horse anti-mouse IgG antibody (1:5000, Cell Signaling Technology), goat anti-rabbit IgG antibody (1:5000, Cell Signaling Technology), and goat anti-chicken (1:3000, Invitrogen). For semiquantification, the band densities were measured using ImageJ software. Uncropped gels and blots that are the most important to our conclusions are provided in the Source Data as a Source Data file.

Statistical analysis. Data were shown as mean \pm SD from at least three separate experiments and/or at least three biologically independent samples. Differences between two data sets were determined by Student $t$-tests (two-sided), and differences for the tumor-free rates were analyzed using the Kaplan-Meier method followed by log-rank statistical test using software SPSS v17.0. Differences were considered significant when $P$ value is less than 0.05 .

Reporting summary. Further information on research design is available in the Nature Research Reporting Summary linked to this article.

\section{Data availability}

All the data generated or analyzed during this study are included in this published article and its supplementary information files. The source data on ENO-1 expression in human bladder cancer cohorts shown in Supplementary Fig. 8 are available in a public repository from the https://www.cbioportal.org/ website. MD simulation data of three protein complexes are available at https://www.nyu.edu/projects/yzhang/MD_data. Source data are provided with this paper.

Received: 18 February 2020; Accepted: 11 March 2021; Published online: 06 April 2021

\section{References}

1. Gil, J. \& Peters, G. Regulation of the INK4b-ARF-INK4a tumour suppressor locus: all for one or one for all. Nat. Rev. Mol. Cell Biol. 7, 667-677 (2006).
2. Park, B. H. \& Vogelstein, B. in Holland-Frei Cancer Medicine (eds. Kufe, D. W. et al.) (BC Decker, 2003).

3. Serrano, M. et al. Role of the INK4a locus in tumor suppression and cell mortality. Cell 85, 27-37 (1996).

4. Lundberg, A. S., Hahn, W. C., Gupta, P. \& Weinberg, R. A. Genes involved in senescence and immortalization. Curr. Opin. Cell Biol. 12, 705-709 (2000).

5. Worsham, M. J. et al. High-resolution mapping of molecular events associated with immortalization, transformation, and progression to breast cancer in the MCF10 model. Breast Cancer Res. Treat. 96, 177-186 (2006).

6. Loughran, O., Edington, K. G., Berry, I. J., Clark, L. J. \& Parkinson, E. K. Loss of heterozygosity of chromosome $9 \mathrm{p} 21$ is associated with the immortal phenotype of neoplastic human head and neck keratinocytes. Cancer Res. 54, 5045-5049 (1994).

7. Wu, X. R. Urothelial tumorigenesis: a tale of divergent pathways. Nat. Rev. Cancer 5, 713-725 (2005).

8. Hustinx, S. R. et al. Concordant loss of MTAP and p16/CDKN2A expression in pancreatic intraepithelial neoplasia: evidence of homozygous deletion in a noninvasive precursor lesion. Mod. Pathol. 18, 959-963 (2005).

9. Kim, W. Y. \& Sharpless, N. E. The regulation of INK4/ARF in cancer and aging. Cell 127, 265-275 (2006).

10. Gonzalez-Zulueta, M. et al. Methylation of the 5' CpG island of the p16/ CDKN2 tumor suppressor gene in normal and transformed human tissues correlates with gene silencing. Cancer Res. 55, 4531-4535 (1995).

11. Robertson, A. G. et al. Comprehensive molecular characterization of muscleinvasive bladder cancer. Cell 171, 540-556.e525 (2017).

12. Serrano, M. The INK4a/ARF locus in murine tumorigenesis. Carcinogenesis 21, 865-869 (2000).

13. Rivandi, M. et al. The $9 \mathrm{p} 21$ locus: a potential therapeutic target and prognostic marker in breast cancer. J. Cell Physiol. 233, 5170-5179 (2018).

14. $\mathrm{Yu}, \mathrm{W}$. et al. Epigenetic silencing of tumour suppressor gene p15 by its antisense RNA. Nature 451, 202-206 (2008).

15. Cunnington, M. S., Santibanez Koref, M., Mayosi, B. M., Burn, J. \& Keavney, B. Chromosome 9p21 SNPs associated with multiple disease phenotypes correlate with ANRIL expression. PLoS Genet. 6, e1000899 (2010).

16. Seoane, J. et al. TGFbeta influences Myc, Miz-1 and Smad to control the CDK inhibitor p15INK4b. Nat. Cell Biol. 3, 400-408 (2001).

17. Hannon, G. J. \& Beach, D. p15INK4B is a potential effector of TGF-betainduced cell cycle arrest. Nature 371, 257-261 (1994).

18. Krimpenfort, P. et al. p15ink $4 \mathrm{~b}$ is a critical tumour suppressor in the absence of p16ink4a. Nature 448, 943-946 (2007).

19. Mo, L. et al. Hyperactivation of Ha-ras oncogene, but not Ink4a/Arf deficiency, triggers bladder tumorigenesis. J. Clin. Invest. 117, 314-325 (2007).

20. Baig, M. H. et al. Enzyme targeting strategies for prevention and treatment of cancer: implications for cancer therapy. Semin. Cancer Biol. 56, 1-11 (2019).

21. McPherson, R. et al. A common allele on chromosome 9 associated with coronary heart disease. Science 316, 1488-1491 (2007).

22. Shea, J. et al. Comparing strategies to fine-map the association of common SNPs at chromosome 9p21 with type 2 diabetes and myocardial infarction. Nat. Genet. 43, 801-805 (2011).

23. Jeck, W. R., Siebold, A. P. \& Sharpless, N. E. Review: a meta-analysis of GWAS and age-associated diseases. Aging Cell 11, 727-731 (2012).

24. Balazs, M., Carroll, P., Kerschmann, R., Sauter, G. \& Waldman, F. M. Frequent homozygous deletion of cyclin-dependent kinase inhibitor 2 (MTS1, p16) in superficial bladder cancer detected by fluorescence in situ hybridization. Genes Chromosomes Cancer 19, 84-89 (1997).

25. Cairns, J. P., Chiang, P. W., Ramamoorthy, S., Kurnit, D. M. \& Sidransky, D. A comparison between microsatellite and quantitative PCR analyses to detect frequent p16 copy number changes in primary bladder tumors. Clin. Cancer Res. 4, 441-444 (1998).

26. Orlow, I. et al. Deletion of the p16 and p15 genes in human bladder tumors [see comments]. J. Natl. Cancer Inst. 87, 1524-1529 (1995).

27. Zhang, Z. T. et al. Role of Ha-ras activation in superficial papillary pathway of urothelial tumor formation. Oncogene 20, 1973-1980 (2001).

28. Jimenez, M. et al. The Rgr oncogene induces tumorigenesis in transgenic mice. Cancer Res. 64, 6041-6049 (2004).

29. Steinberg, G. D. Bladder cancer: assessing the conundrum of microscopic haematuria. Nat. Rev. Urol. 13, 700-701 (2016).

30. Mertens, L. S., Neuzillet, Y., Horenblas, S. \& van Rhijn, B. W. Landmarks in non-muscle-invasive bladder cancer. Nat. Rev. Urol. 11, 476-480 (2014).

31. Dinney, C. P. et al. Focus on bladder cancer. Cancer Cell 6, 111-116 (2004).

32. Grossman, H. B. Superficial bladder cancer: decreasing the risk of recurrence. Oncology 10, 1617-1624 (1996).

33. Sharpless, N. E. et al. Loss of p16Ink4a with retention of p19Arf predisposes mice to tumorigenesis. Nature 413, 86-91 (2001).

34. $\mathrm{Wu}, \mathrm{X}$. R. Biology of urothelial tumorigenesis: insights from genetically engineered mice. Cancer Metastasis Rev. 28, 281-290 (2009).

35. Van Batavia, J. et al. Bladder cancers arise from distinct urothelial subpopulations. Nat. Cell Biol. 16, 982-991 (2014). 
36. Papafotiou, G. et al. KRT14 marks a subpopulation of bladder basal cells with pivotal role in regeneration and tumorigenesis. Nat. Commun. 7, 11914 (2016).

37. Earl, J. et al. The UBC- 40 urothelial bladder cancer cell line index: a genomic resource for functional studies. BMC Genomics 16, 403 (2015).

38. Kent, L. N. \& Leone, G. The broken cycle: E2F dysfunction in cancer. Nat. Rev. Cancer 19, 326-338 (2019).

39. Malumbres, M. \& Barbacid, M. Cell cycle, CDKs and cancer: a changing paradigm. Nat. Rev. Cancer 9, 153-166 (2009).

40. Russo, A. A., Tong, L., Lee, J. O., Jeffrey, P. D. \& Pavletich, N. P. Structural basis for inhibition of the cyclin-dependent kinase Cdk6 by the tumour suppressor p16INK4a. Nature 395, 237-243 (1998).

41. Hedegaard, J. et al. Comprehensive transcriptional analysis of early-stage urothelial carcinoma. Cancer Cell 30, 27-42 (2016).

42. Ahmad, I. et al. K-Ras and beta-catenin mutations cooperate with Fgfr3 mutations in mice to promote tumorigenesis in the skin and lung, but not in the bladder. Dis. Model. Mech. 4, 548-555 (2011).

43. Zhou, H. et al. FGFR3b extracellular loop mutation lacks tumorigenicity in vivo but collaborates with $\mathrm{p} 53 / \mathrm{pRB}$ deficiency to induce high-grade papillary urothelial carcinoma. Sci. Rep. 6, 25596 (2016).

44. Herman, J. G. et al. Distinct patterns of inactivation of p15INK4B and p16INK4A characterize the major types of hematological malignancies. Cancer Res. 57, 837-841 (1997).

45. Iravani, M., Dhat, R. \& Price, C. M. Methylation of the multi tumor suppressor gene-2 (MTS2, CDKN1, p15INK4B) in childhood acute lymphoblastic leukemia. Oncogene 15, 2609-2614 (1997).

46. Bayley, J. P. \& Devilee, P. The Warburg effect in 2012. Curr. Opin. Oncol. 24, 62-67 (2012).

47. Sanchez-Carbayo, M., Socci, N. D., Lozano, J., Saint, F. \& Cordon-Cardo, C. Defining molecular profiles of poor outcome in patients with invasive bladder cancer using oligonucleotide microarrays. J. Clin. Oncol. 24, 778-789 (2006).

48. Lee, J. S. et al. Expression signature of E2F1 and its associated genes predict superficial to invasive progression of bladder tumors. J. Clin. Oncol. 28 , 2660-2667 (2010).

49. van Kessel, K. E., Zuiverloon, T. C., Alberts, A. R., Boormans, J. L. \& Zwarthoff, E. C. Targeted therapies in bladder cancer: an overview of in vivo research. Nat. Rev. Urol. 12, 681-694 (2015).

50. Zivotic, I. et al. CDKN2B gene expression is affected by 9 p21.3 rs 10757278 in CAD patients, six months after the MI. Clin. Biochem. 73, 70-76 (2019).

51. Helgadottir, A. et al. A common variant on chromosome 9p21 affects the risk of myocardial infarction. Science 316, 1491-1493 (2007)

52. Aguilo, F., Zhou, M. M. \& Walsh, M. J. Long noncoding RNA, polycomb, and the ghosts haunting INK4b-ARF-INK4a expression. Cancer Res. 71, 5365-5369 (2011).

53. Latres, E. et al. Limited overlapping roles of P15(INK4b) and P18(INK4c) cell cycle inhibitors in proliferation and tumorigenesis. EMBO J. 19, 3496-3506 (2000).

54. Eswar, N. et al. Comparative protein structure modeling using Modeller. Curr. Protoc. Bioinformatics. Chapter 5, Unit-5.6 (2006).

55. Jeffrey, P. D., Tong, L. \& Pavletich, N. P. Structural basis of inhibition of CDKcyclin complexes by INK4 inhibitors. Genes Dev. 14, 3115-3125 (2000).

56. Yang, J. et al. The I-TASSER Suite: protein structure and function prediction. Nat. Methods 12, 7-8 (2015).

57. Roy, A., Kucukural, A. \& Zhang, Y. I-TASSER: a unified platform for automated protein structure and function prediction. Nat. Protoc. 5, 725-738 (2010).

58. Yuan, C., Li, J., Selby, T. L., Byeon, I. J. \& Tsai, M. D. Tumor suppressor INK4: comparisons of conformational properties between p16(INK4A) and p18 (INK4C). J. Mol. Biol. 294, 201-211 (1999).

59. Maier, J. A. et al. ff14SB: improving the accuracy of protein side chain and backbone parameters from ff99SB. J. Chem. Theory Comput. 11, 3696-3713 (2015).

60. Case, D. et al. AMBER16, Technical report, San Francisco (2016).

61. Roe, D. R. \& Cheatham, T. E. 3rd PTRAJ and CPPTRAJ: software for processing and analysis of molecular dynamics trajectory data. J. Chem. Theory Comput. 9, 3084-3095 (2013).

62. Darden, T., York, D. \& Pedersen, L. Particle mesh Ewald: an N. $\log (\mathrm{N})$ method for Ewald sums in large systems. J. Chem. Phys. 98, 10089-10092 (1993).

63. Andersen, H. C. Rattle: A "velocity" version of the shake algorithm for molecular dynamics calculations. J. Comp. Phys. 52, 24-34 (1983).
64. Ester, M., Kriegel, H.-P., Sander, J. \& Xu X. A density-based algorithm for discovering clusters in large spatial databases with noise. In Kdd, 1996, Vol. 96, 226-231 (AAAI Press, 1996).

65. O'Farrell, P. H. High resolution two-dimensional electrophoresis of proteins. J. Biol. Chem. 250, 4007-4021 (1975).

66. Burgess-Cassler, A., Johansen, J. J., Santek, D. A., Ide, J. R. \& Kendrick, N. C. Computerized quantitative analysis of coomassie-blue-stained serum proteins separated by two-dimensional electrophoresis. Clin. Chem. 35, 2297-2304 (1989).

67. Jafari, M. et al. Comparison of in-gel protein separation techniques commonly used for fractionation in mass spectrometry-based proteomic profiling. Electrophoresis 33, 2516-2526 (2012).

68. Larkin, M. A. et al. Clustal W and Clustal X version 2.0. Bioinformatics 23, 2947-2948 (2007)

\section{Acknowledgements}

We wish to acknowledge Lijie Ma and Fengxia Zhang for their excellent technical assistance with some of the histopathological analysis. This work was supported in part by National Institutes of Health (P01 CA165980) and awards from the United States Veterans Affairs Office of Research and Development (Biomedical Laboratory Research and Development Service: Merit Review (1I01BX002049) and Research Career Scientist (1IK6BX004479)). Y.Z. wishes to acknowledge the funding from the National Institute of General Medical Sciences (R35 GM127040).

\section{Author contributions}

The first three authors contributed equally to this work. Y.X. designed and carried out the initial in vitro and in vivo experiments, organized the data, and prepared the figures; Y.L. designed and conducted all the in vitro and in vivo experiments during revision; C.Y. and Y.Z. performed the structural analyses and modeling; D.M.S., T.T.S., D.J.D., and M.S.T. contributed to data analysis and interpretation and manuscript editing; X.R.W. conceived the study, helped design the experiments and interpret the data, and wrote and revised the manuscript.

\section{Competing interests}

The authors declare no competing interests.

\section{Additional information}

Supplementary information The online version contains supplementary material available at https://doi.org/10.1038/s41467-021-22327-5.

Correspondence and requests for materials should be addressed to X.-R.W.

Peer review information Nature Communications thanks Tatu Pantsar, David Santamaría and the other, anonymous, reviewer(s) for their contribution to the peer review of this work. Peer reviewer reports are available.

Reprints and permission information is available at http://www.nature.com/reprints

Publisher's note Springer Nature remains neutral with regard to jurisdictional claims in published maps and institutional affiliations.

Open Access This article is licensed under a Creative Commons Attribution 4.0 International License, which permits use, sharing, adaptation, distribution and reproduction in any medium or format, as long as you give appropriate credit to the original author(s) and the source, provide a link to the Creative Commons license, and indicate if changes were made. The images or other third party material in this article are included in the article's Creative Commons license, unless indicated otherwise in a credit line to the material. If material is not included in the article's Creative Commons license and your intended use is not permitted by statutory regulation or exceeds the permitted use, you will need to obtain permission directly from the copyright holder. To view a copy of this license, visit http://creativecommons.org/ licenses/by/4.0/.

(C) The Author(s) 2021 